\title{
Operating under jet splashing conditions can increase the capture efficiency of scoops
}

\author{
A. Kruisbrink, P. Paleo Cageao*, H.P. Morvan, K. Simmons \\ Gas Turbine and Transmissions Research Centre (G2TRC) \\ Faculty of Engineering, University of Nottingham, UK
}

\begin{abstract}
Scoops are used in aero-engines to capture oil and direct the oil flow for the lubrication of bearings, where a direct oil injection is not effective or possible. The design of scoops focuses on the capture of oil to aim for the highest capture efficiency. The operating conditions are usually chosen such that splashing of the oil jet against the outer side of scoops is avoided. In this paper it is shown that some degree of splashing may be beneficial and results in an increase of the scoop capture efficiency. An analytical approach is presented to describe the range of operating conditions at which the jet is aimed to hit a specific fixed point on the outer or inner scoop contour. These operating conditions are introduced as splashing and capture conditions, respectively, and described in terms of a dimensionless velocity ratio and the jet angle. A "splashing criterion" is introduced to describe the operating conditions at which the jet is aimed to hit the rear side of the scoop. A "capture criterion" is introduced to describe the operating conditions at which the jet is aimed to hit the scoop tip. The basic assumption in this paper is that the best scoop capture efficiency is correlated to the splashing criterion and not to the capture criterion. The correlation is confirmed by a series of experiments on a scoop, performed at different jet angles and a range of velocity ratios. From the match of the correlation curve with the experiments, it is concluded that the best scoop efficiency is obtained when the jet is aimed to splash on the outer scoop contour at a point near the rear side. It could be proven that this splash point does not change with the jet angle. The fact that this point of "best efficiency" remains a fixed splashing point on the scoop contour may be helpful in the design of scoops.
\end{abstract}

Keywords: Aero-engine, Gas Turbine, Lubrication, Scoop Capture efficiency, Analytical, Experimental.

\section{Introduction}

In aero-engines jet lubrication is commonly used to lubricate and cool its transmission components, such as bearings and gears. For rotational shaft speeds up to $15000 \mathrm{rpm}$, the oil jet is usually directed into the annular gap between the inner and outer race [1], as illustrated in Fig. 1 (left). As shaft speeds are pushed upwards, conventional jet lubrication fails to adequately lubricate and cool the inner-race contact, since the oil is thrown outwards due to centrifugal effects. Then, a more effective and efficient means of lubrication, known as under-race lubrication, is preferred [1, 2, 3, 4]. Figure 1 (right) shows a side feed underrace lubrication, used to direct oil under the inner race, which is centrifugally distributed through a plurality of holes in the inner race, to lubricate and cool the bearing [1].

When under-race lubrication is required, but there is no access for side feed under the inner race, the oil can be directed inward through slots in the rotor or shaft using a rotating "scoop" device. Oil from one or more injectors is directed towards a sharp-edged scoop, captured as the shaft rotates and directed inward through slots in the shaft, as shown in Fig. 2. The oil is injected under an angle, which is prescribed by the nozzle orientation. The oil is then guided in the axial direction along

\footnotetext{
${ }^{*}$ Corresponding author

Email address: Paloma.Paleo@nottingham.ac.uk (P. Paleo Cageao)
}

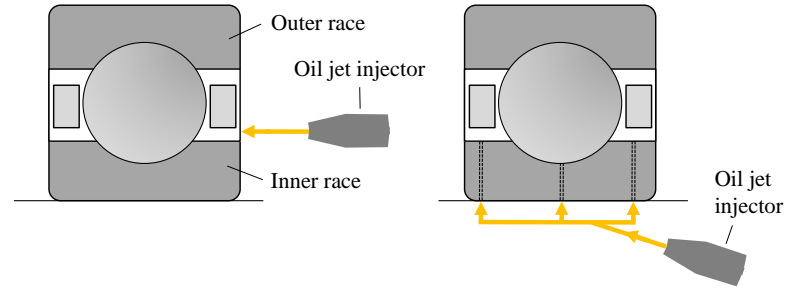

Figure 1: Lubrication of high speed bearing: (left) conventional jet lubrication and (right) side feed under-race lubrication.

passages and delivered to the bearings. The oil trajectory is illustrated in Fig. 2 and Fig. 3.

The concept of scoops for oil distribution was introduced in the 1970's. Brown [3] describes an under-race lubrication system for main shaft bearings on turbo fan engines which includes an axial scoop system. The oil jet is targeted axially at a passage that directs the oil under, and centrifugally out through holes in, the inner race to cool and lubricate the bearing.

\subsection{Scoop design}

An early scoop design for a gas turbine shaft is described in the 1987 patent of Koveleski [5]. The invention is a scoop system that directs specific proportions of oil axially, forward and rearward from the scoop to lubricate bearings located on opposite sides of the scoop itself, along the shaft. The author 


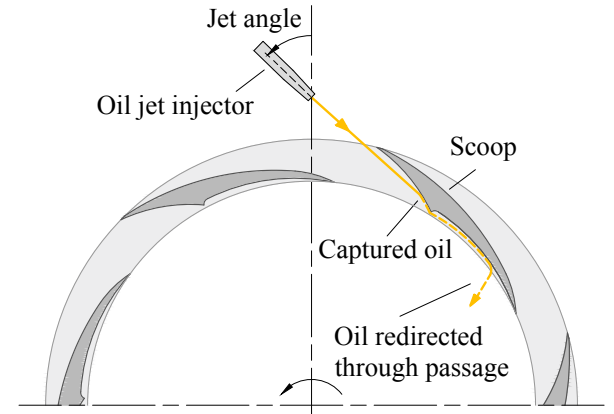

Figure 2: Principle of scoop operation. Oil trajectory before capture (yellow line) and after capture (dashed yellow line).

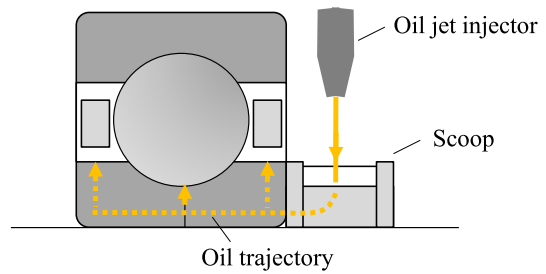

Figure 3: Under-race lubrication via scoop.

presented a scoop having an axially extending ridge at the beginning of the passage, which serves as a dam to suppress the oil spillage due to centrifugal forces. The number of scoops in the patent was four. Over the years, scoop design has evolved in order to meet the increasingly demanding requirements of gas turbines for higher stage loadings and consequently higher operating speeds. The patents of Fisher et al [6], with three scoop blades, and Fisher [7], with four scoop blades, are for conventional flat bladed scoops, whereas that of Dins et al [8], with scoops that may comprise one blade, two, three or more blades, describes a scoop with a curved blade, intended to maximize fluid capture. In $2016 \mathrm{McDonagh}$ [9] patented the concept of a bi-directional scoop, with four inlet openings, where the key difference to previously patented scoop designs is that the oil trajectory through the scoop follows an angled path such that it is turning throughout its journey rather than travelling first perpendicular to the shaft and then parallel to it.

Efforts to improve the scoop system have been, to date, focused on the oil capture. Key geometrical characteristics are related to the inner scoop contour (e.g., flat blades [5, 6, 7], curved blades [8] or skewed blades [9]) and to the constriction at the end of the blades, to prevent oil from flowing back $[5,7,8,9]$.

\subsection{Research on scoops}

Experimental research has recently been conducted at the Gas Turbine and Transmissions Research Centre (G2TRC) of the University of Nottingham to investigate the capture efficiency of a scoop oil lubrication system [10]. Variations of the scoop geometry, jet configuration and jet angle were investigated over a range of operating conditions. Cageao et al. [10] have shown that the overall capture efficiency not only depends on the amount of oil captured by the scoop, but also on how much is retained. A key parameter is the oil jet angle which should be considered in the design of a scoop feed system (see Fig. 2). At specific velocity ratio, lower angles seem to improve the capture efficiency of the scoop, even if the oil jet may hit the outer surface of the scoop. A theoretical efficiency characteristic is introduced as function of the velocity ratio. At velocity ratios lower than a critical value the oil jet is not fully captured, which results in the reduction of the efficiency. In the present paper, this critical velocity ratio is introduced as "capture criterion".

Several numerical methods are available for the modelling of two-phase flows. Most commonly used are mesh-based methods like conventional Computational Fluid Dynamics (CFD), with Volume of Fluid (VOF), level-set or front tracking methods to evolve the interface. Few literature is found about Direct Numerical Simulation (DNS) [11]. Ma et al. [12, 13] use DNS in statistical learning. The results of DNS simulations were used as input for Neural Networks, to obtain closure relations for the averaged two-fluid equations, in the application of bubbly flows. Alternatives are mesh-less methods such as Smoothed Particle Hydrodynamics (SPH) [14, 15, 16]. Further can be mentioned the combination of front tracking and ghost fluid methods, where moving surfaces are tracked by marker particles [17]. Some literature is found on two-phase jet flows $[14,17]$, although few literature includes the droplet formation around scoops [16].

Published numerical investigations on scoops in aero-engine applications are:

Prasad et al. [18] investigated a scoop configuration involving two scoops and two nozzles. The paper gives very little geometric data but the scoop tip is sharp edged. CFD simulations with the multiphase VOF method were performed to investigate the effect of parameters such as the oil jet velocity, shaft speed, scoop width and outer diameter. No experimental data was included in the paper, although the authors state that the CFD results agree well within $2 \%$ of the test results.

Korsukova et al. [16] conducted a numerical investigation using both conventional CFD and SPH on a geometry very similar to that investigated experimentally in [10]. The 2-D extrusion and 3-D models provide significant insight into flow behaviour near the scoops and the paper highlights key features which were also seen experimentally, such as the formation of plumes, droplets and ligaments. The numerical data is compared with the experiments showing a good qualitative agreement of the capture efficiency and a reasonable quantitative agreement within $10 \%$.

Prabhakar et al. [19] evaluated the capture efficiency using conventional CFD. Two parameters were investigated in an attempt to reduce the plume formation and to improve the capture efficiency. The authors claim that the capture efficiency increases about $2 \%$ by blunting the tip of the edge of the scoop. However, targeting the oil with shaft rotation reduces the capture efficiency by $10 \%$, compared to targeting against shaft rotation.

Yaguo et al. [20] validated the capture efficiency obtained 
with conventional CFD against experiments, showing an agreement within $14 \%$. The effect of the shaft speed, jet velocity and number of nozzles (up to three) was studied. The capture efficiency of the radial scoop design was rather low (max. 60\%).

The oil scoops systems are not $100 \%$ efficient. Visualization tests show that efficiency losses may arise due to: i) the formation of plumes, ligaments and droplets after scoop slicing, which are not captured by the next scoop, and ii) part of the oil initially captured by the scoop is accelerated outwards through centrifugal forces and eventually leaves the scoop [10].

The operating conditions of a scoop are described by a dimensionless velocity ratio and the jet angle. The velocity ratio is defined as the ratio of scoop tip velocity and jet velocity

$$
V R=\frac{V_{t i p}}{V_{\text {jet }}}=\frac{\omega R_{t i p}}{V_{\text {jet }}},
$$

where $V_{\text {tip }}$ is the scoop tip velocity, $V_{\text {jet }}$ the jet velocity, $\omega$ the rotational speed of the shaft and $R_{\text {tip }}$ the scoop tip radius.

In this paper an analytical method is described, which predicts the range of operating conditions under which splashing and capturing takes place. It is shown that the best capture efficiency is obtained when the oil jet is aimed to hit the outer surface of the scoop near the rear side. The best efficiency appears to be correlated to a "splashing criterion", which is introduced and formulated in Section 2. The operating conditions for oil jet contact with the inner scoop surface are described by a "capture criterion", as formulated in Section 3. Experiments on a scoop configuration are described in Section 4, and used to validate the analytical method in Section 5. A few applications are included in Section 6, where the effect of the jet diameter, a conical jet or plume and the number of scoops on the splashing and capture criteria is investigated, as well as scaling to other scoop configurations. The analytical method may be used in the design of the scoop geometry and the specification of its operating conditions. The method provides the operating conditions for the best efficiency, although it does not provide the magnitude of the best efficiency.

The analytical model is based on geometric [L, T] conditions. Dynamic effects [M,L,T], such as shear and surface tension forces that may lead to the formation of plumes and droplets, are not taking into account in the splashing and capture conditions and criteria.

\section{Splashing criterion}

In this section splashing of the oil jet against the outer scoop contour is considered. A special case is introduced at which the jet is aimed to hit the rear side of the scoop.

Consider a jet, which is directed to one of the scoops. The jet hits and is sliced by the scoop tip (Fig. 4: left). After this instant, the jet is assumed to grow linear in time with the jet velocity and aimed to hit an arbitrary point on the outer contour of the scoop (Fig. 4: right). This operating condition is introduced as a splashing condition. It is assumed that the jet does not hit the outer scoop contour at any other point during its growth (see further analysis in Section 2.1). The outer scoop contour is described by the radius $R_{o}(\alpha)$ as function of the scoop angle $\alpha$, within the range $0 \leq \alpha \leq \alpha_{\text {scoop }}$ from scoop tip ( $\alpha=0$ ) to rear $\left(\alpha=\alpha_{\text {scoop }}\right)$. The motion of the scoop is described in cylindrical coordinates $[R, \theta]$. The jet position is described by the radius $R_{\text {jet }}$ on the vertical axis, and its direction by the jet angle $\phi_{\text {jet }}$.
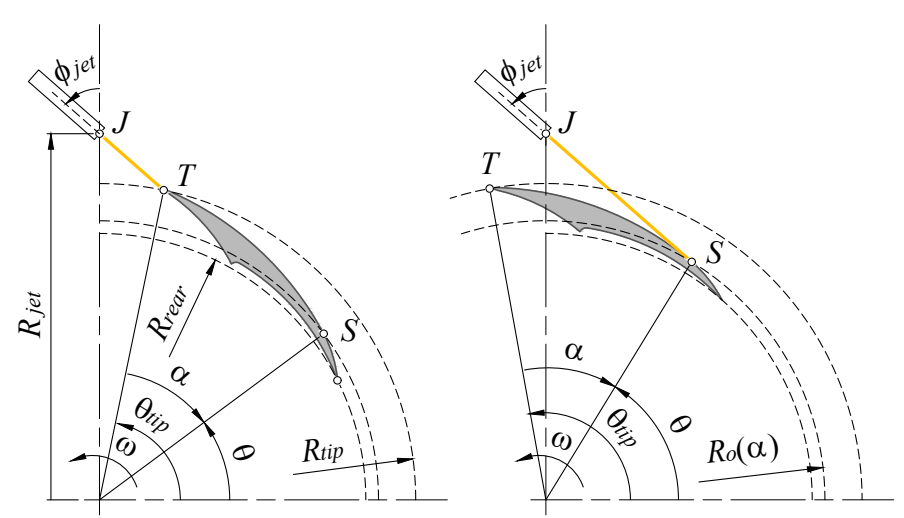

Figure 4: Initial slicing condition at instant $t_{i}$ (left) and final splashing condition at instant $t_{f}$ (right).

To describe the above splashing conditions, the dimensionless velocity ratio in Eq. (1) is rewritten as

$$
V R=\frac{\omega\left(t_{f}-t_{i}\right) R_{t i p}}{V_{j e t}\left(t_{f}-t_{i}\right)}=\frac{\left[\theta\left(t_{f}\right)-\theta\left(t_{i}\right)\right] R_{t i p}}{d_{J S}-d_{J T}},
$$

where $t_{i}$ and $t_{f}$ are the instant of initial slicing and final splashing respectively, $\theta\left(t_{f}\right)-\theta\left(t_{i}\right)$ is the angle over which the scoop has rotated, and $\left(d_{J S}-d_{J T}\right)$ the distance over which the jet has travelled from scoop tip (point $T$ ) to splashing point (point $S$ ). The initial slicing conditions $\left(t=t_{i}\right)$ are first evaluated and then the final splashing conditions $\left(t=t_{f}\right)$.

The initial angular position of the scoop tip (point $T$ ), written as function of the jet angle, is described by (Fig. 4: left)

$$
\cos \left(\theta_{t i p}\left(t_{i}\right)\right)=\frac{d_{J T}}{R_{t i p}} \sin \left(\phi_{j e t}\right),
$$

where the distance from injector (point $\mathbf{J}$ ) to scoop tip is

$$
d_{J T}=R_{\text {jet }}\left\{\cos \left(\phi_{j e t}\right)-\sqrt{\left(\frac{R_{t i p}}{R_{\text {jet }}}\right)^{2}-\sin ^{2}\left(\phi_{j e t}\right)}\right\} .
$$

The initial angular position of the splashing point (point $S$ ) now becomes

$$
\theta\left(t_{i}\right)=\theta_{t i p}\left(t_{i}\right)-\alpha,
$$

where $\alpha$ is the angle between scoop tip and splashing point. 
The final angular position of the splashing point, also written as function of the jet angle, is (Fig. 4: right)

$$
\cos \left(\theta\left(t_{f}\right)\right)=\frac{d_{J S}}{R_{o}(\alpha)} \sin \left(\phi_{j e t}\right),
$$

where the distance from injector to splashing point is

$$
d_{J S}=R_{j e t}\left\{\cos \left(\phi_{j e t}\right)-\sqrt{\left(\frac{R_{o}(\alpha)}{R_{j e t}}\right)^{2}-\sin ^{2}\left(\phi_{j e t}\right)}\right\}
$$

From the above equation it follows for the range of jet angles

$$
\phi_{j e t}<\sin ^{-1}\left(\frac{R_{o}(\alpha)}{R_{j e t}}\right)
$$

With the above equations the initial and final positions of the scoop are described. The scoop has rotated over an angle

$$
\theta\left(t_{f}\right)-\theta\left(t_{i}\right)=\theta\left(t_{f}\right)-\theta_{t i p}\left(t_{i}\right)+\alpha .
$$

The distance over which the jet front has travelled is $\left(d_{J S}-d_{J T}\right)$, so that the time interval needed for travelling becomes

$$
t_{f}-t_{i}=\frac{d_{J S}-d_{J T}}{V_{j e t}} .
$$

Substitution of the Eqs. (3), (6) and (9) in Eq. (2) finally yields

$$
V R_{\text {splash }}=\frac{\left[\cos ^{-1}\left\{\frac{d_{J S} \sin \left(\phi_{j e t}\right)}{R_{o}(\alpha)}\right\}-\cos ^{-1}\left\{\frac{d_{J T} \sin \left(\phi_{j e t}\right)}{R_{t i p}}\right\}+\alpha\right] R_{t i p}}{d_{J S}-d_{J T}} .
$$

where the distances $d_{J S}$ and $d_{J T}$ are given in the Eqs. (4) and (7), respectively. The above result describes the splashing conditions of the jet when hitting a specific point $\left[\alpha, R_{o}(\alpha)\right]$ on the outer scoop contour, written in terms of a velocity ratio, which in its turn is a function of the jet angle $\phi_{j e t}$ and scoop angle $\alpha$.

The splashing condition at which the jet is aimed to hit or splash on the rear side of the scoop is a special case, introduced as the "splashing criterion". The splashing criterion is obtained by substituting $\alpha=\alpha_{\text {scoop }}$ and $R_{o}(\alpha)=R_{\text {rear }}$ in the above equation.

$V R_{\text {splash }}^{*}=\frac{\left[\cos ^{-1}\left\{\frac{d_{J S} \sin \left(\phi_{j e t}\right)}{R_{\text {rear }}}\right\}-\cos ^{-1}\left\{\frac{d_{J T} \sin \left(\phi_{j e t}\right)}{R_{t i p}}\right\}+\alpha_{\text {scoop }}\right] R_{\text {tip }}}{d_{J S}-d_{J T}}$.

An example of splashing conditions and the splashing criterion is given in Fig. 5.

The maximum jet angle follows from Eq. (8). Above this angle the jet no longer hits the outer scoop contour.

In Section 6, applications of the splashing criterion and splashing conditions are shown for some scoop configurations.
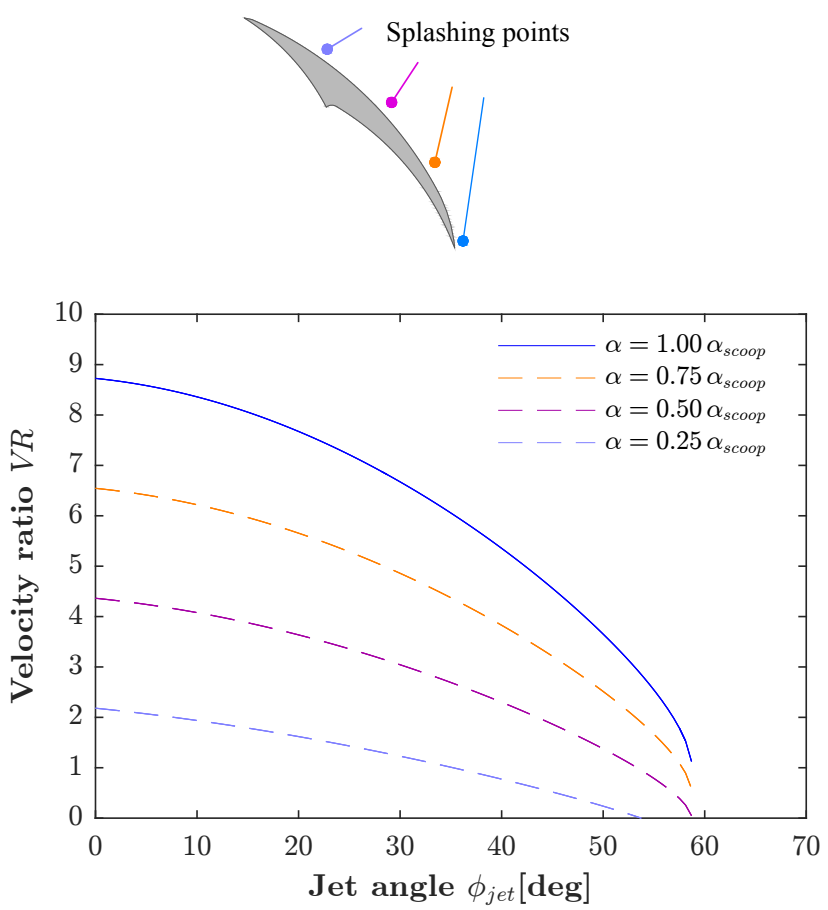

Figure 5: Scoop operating characteristic. Splashing criterion (blue line) with splashing point on rear side. Splashing conditions (dashed lines) with splashing points on outer contour.

\subsection{Contour of jet front}

In this section the contour of the jet front is described under splashing criterion conditions.

After slicing by the scoop tip, the jet grows linearly in time with the jet velocity along a straight line. Here we describe the non-linear contour of the jet front relative to the (outer) scoop contour. For this purpose the jet is assumed to rotate around the stagnant scoop with rotational shaft speed $-\omega$, as shown in Fig. 6.

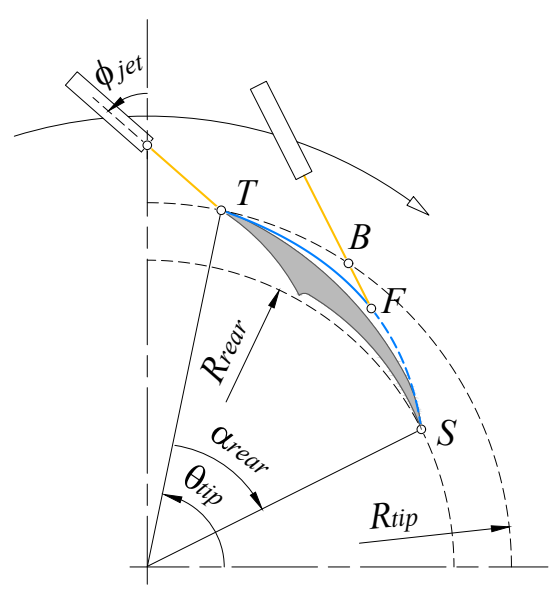

Figure 6: Contour of jet relative to scoop contour. The jet hits the scoop tip (point T), grows in time with moving jet front (point $\mathrm{F}$ ) and splashes (point $\mathrm{S}$ ) on outer scoop contour. 
The jet hits the scoop tip (point $T$ ) at the instant $t=t_{i}$. From this point on the rotation of the jet front (point F) around the scoop is described by $\left(t<t_{c r}\right)$

$$
\begin{gathered}
{\left[\begin{array}{l}
x_{B} \\
y_{B}
\end{array}\right]=R_{t i p}\left[\begin{array}{c}
\cos \left(\theta_{\text {tip }}-\omega\left(t-t_{i}\right)\right) \\
\sin \left(\theta_{\text {tip }}-\omega\left(t-t_{i}\right)\right)
\end{array}\right] .} \\
{\left[\begin{array}{l}
x_{F} \\
y_{F}
\end{array}\right]=\left[\begin{array}{l}
x_{B} \\
y_{B}
\end{array}\right]+d_{B F}\left[\begin{array}{c}
\cos \left(\phi_{\text {jet }}-\pi / 2-\omega\left(t-t_{i}\right)\right) \\
\sin \left(\phi_{\text {jet }}-\pi / 2-\omega\left(t-t_{i}\right)\right)
\end{array}\right] .}
\end{gathered}
$$

The first equation describes the rotation of basic jet point B, moving from point $\mathrm{T}$ along a virtual circle with radius equal to the scoop tip radius. The second equation describes the position of point $B$ plus the change of jet direction and its growth, represented by the jet length $d_{B F}$. As a next step the length of the growing jet is evaluated.

The splashing criterion is described by the critical velocity ratio, which may be written as

$$
V R_{\text {splash }}=\frac{\omega\left(t-t_{i}\right) R_{t i p}}{V_{j e t}\left(t-t_{i}\right)} .
$$

The jet growth in time is

$$
d_{B F}=V_{j e t}\left(t-t_{i}\right)
$$

Thus follows

$$
d_{B F}=\frac{\omega\left(t-t_{i}\right) R_{t i p}}{V R_{\text {splash }}} .
$$

The jet hits the rear side of the scoop if

$$
d_{B F}=d_{J S}-d_{J T}=\frac{\omega\left(t_{f}-t_{i}\right) R_{t i p}}{V R_{\text {splash }}} .
$$

The contour of the jet front relative to that of the scoop is now described by the Eqs (13), (14) and (17). The final instant $t_{c r}$ is obtained from Eq. (18). The distances $d_{J T}$ and $d_{J S}$ are obtained from Eqs. (4) and (7), respectively. The critical velocity ratio is obtained from Eq. (12) with substitution of $R_{o}(\alpha)=R_{\text {rear }}$. Note that these equations hold for a specific jet angle $\phi_{\text {jet }}$.

The analysis may be used in the design of the scoop contour. If the jet is not supposed to hit the scoop, the outer scoop contour should be shaped such that it does not touch the contour of the jet front. If the jet is supposed to hit a specific point on the outer scoop contour, the scoop profile upstream of this point should not touch the contour of the jet front. In Section 6.5 an application is given of the contour of the jet front together with a scoop profile.

\section{Capture criterion}

In this section we consider the capture of the oil jet by the scoop. The jet may not be fully captured if the shaft speed is relatively low compared to the jet velocity, i.e. at low velocity ratios. A special case is introduced at which the jet is just fully captured.
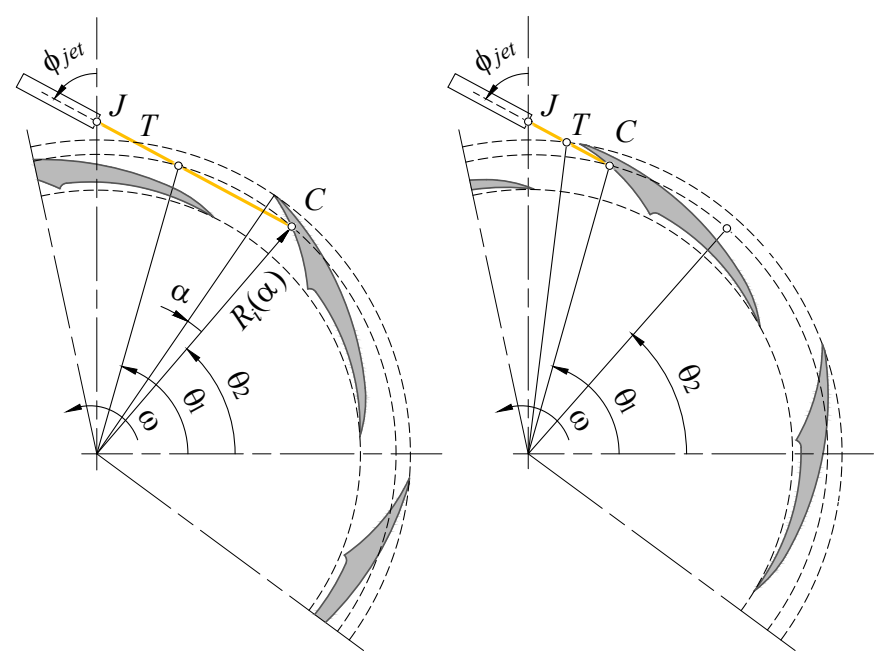

Figure 7: Capture conditions. After being sliced (point $T$ ), the jet hits the inner surface of the next scoop. The two solutions of Eq. (23) are shown in the left and right figure (point $C$ ).
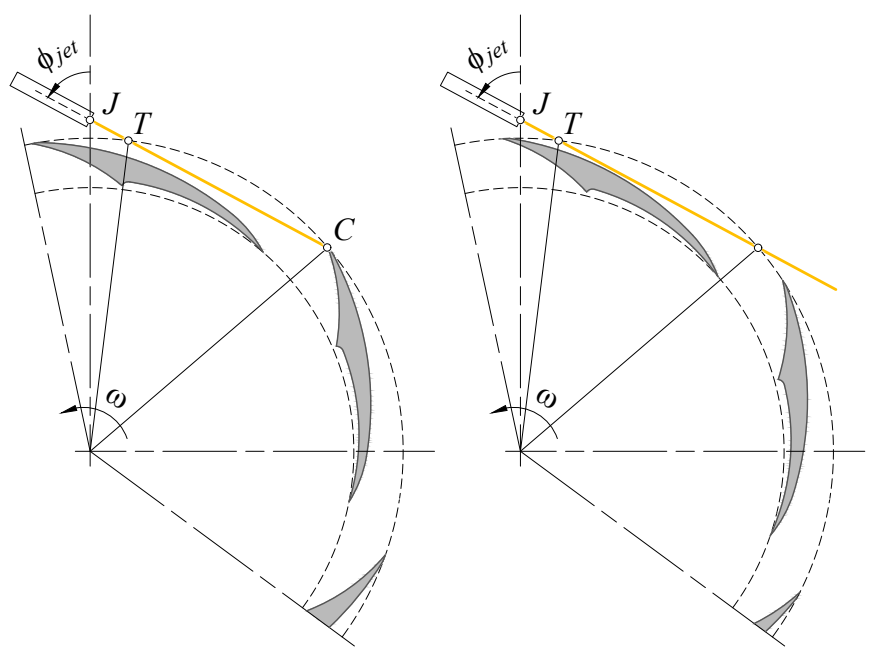

Figure 8: The jet is just fully captured by the scoop tip $\left(V R=V R_{\text {capt }}^{+}\right.$; left $)$. The jet misses the scoop tip and is not fully captured $\left(V R<V R_{\text {capt }}^{+}\right.$; right).

Consider the scoop rotation in Fig. 7. After being sliced by a scoop, the jet is assumed to grow linear in time with the jet velocity, and aimed to hit an arbitrary point on the inner contour of the scoop. This operating condition is introduced as a capture condition. It is again assumed that the jet does not hit the scoop contour at any other point during its growth. The inner scoop contour is described by the radius $R_{i}(\alpha)$ as function of the scoop angle $\alpha$. The initial slicing conditions $\left(t=t_{i}\right)$ are first evaluated and then the final capture conditions $\left(t=t_{f}\right)$.

The initial angular position of the scoop tip (point $T$ ), written as function of the jet angle, is described by the Eqs. (3) and (4). The initial angular position of the capture point (point $\mathrm{C}$ ) on the next scoop now becomes

$$
\theta\left(t_{i}\right)=\theta_{\text {tip }}\left(t_{i}\right)-\alpha-2 \pi / N_{\text {scoops }},
$$


where $\alpha$ is the angle between scoop tip and capture point.

The final angular position of the capture point, also written as function of the jet angle, is (Fig. 7: left and right)

$$
\begin{aligned}
& \cos \left(\theta_{1}\left(t_{f}\right)\right)=\frac{d_{J C}^{-}}{R_{i}(\alpha)} \sin \left(\phi_{j e t}\right) \\
& \cos \left(\theta_{2}\left(t_{f}\right)\right)=\frac{d_{J C}^{+}}{R_{i}(\alpha)} \sin \left(\phi_{j e t}\right)
\end{aligned},
$$

where the distance from injector (point $\mathbf{J}$ ) to capture point (point $C)$ is

$$
\begin{aligned}
& d_{J C}^{-}=R_{\text {jet }}\left\{\cos \left(\phi_{\text {jet }}\right)-\sqrt{\left(\frac{R_{i}(\alpha)}{R_{\text {jet }}}\right)^{2}-\sin ^{2}\left(\phi_{j e t}\right)}\right\} \\
& d_{J C}^{+}=R_{\text {jet }}\left\{\cos \left(\phi_{\text {jet }}\right)+\sqrt{\left(\frac{R_{i}(\alpha)}{R_{\text {jet }}}\right)^{2}-\sin ^{2}\left(\phi_{j e t}\right)}\right\} .
\end{aligned}
$$

Note that there are two solutions. The minus sign represents the relatively small jet growth (Fig. 7: right) and the plus sign the relatively large jet growth (Fig. 7: left). The scoop has rotated over the angles

$$
\begin{gathered}
\theta_{1}\left(t_{f}\right)-\theta\left(t_{i}\right)=\theta_{1}\left(t_{f}\right)-\theta_{\text {tip }}\left(t_{i}\right)+\alpha+2 \pi / N_{\text {scoops }} \\
\theta_{2}\left(t_{f}\right)-\theta\left(t_{i}\right)=\theta_{2}\left(t_{f}\right)-\theta_{\text {tip }}\left(t_{i}\right)+\alpha+2 \pi / N_{\text {scoops }} .
\end{gathered}
$$

Substitution of the Eqs. (3), (4) and (19) to (21) in Eq. (2) finally yields after some manipulation

$\begin{aligned} V R_{\text {capt }}^{-} & =\frac{\left[\cos ^{-1}\left\{\frac{d_{J C}^{-} \sin \left(\phi_{j e t}\right)}{R_{i}(\alpha)}\right\}-\cos ^{-1}\left\{\frac{d_{J T} \sin \left(\phi_{j e t}\right)}{R_{\text {tip }}}\right\}+\alpha+\frac{2 \pi}{N_{\text {scoops }}}\right] R_{\text {tip }}}{d_{J C}^{-}-d_{J T}} \\ V R_{\text {capt }}^{+} & =\frac{\left[\cos ^{-1}\left\{\frac{d_{J C}^{+} \sin \left(\phi_{j e t}\right)}{R_{i}(\alpha)}\right\}-\cos ^{-1}\left\{\frac{d_{J T} \sin \left(\phi_{j e t}\right)}{R_{\text {tip }}}\right\}+\alpha+\frac{2 \pi}{N_{\text {scoops }}}\right] R_{\text {tip }}}{d_{J C}^{+}-d_{J T}}\end{aligned}$

The above result describes the capture conditions of the jet when hitting a specific point $\left[\alpha, R_{i}(\alpha)\right]$ on the inner scoop contour.

The capture condition at which the jet is aimed to hit the scoop tip is a special case, introduced as the "capture criterion", as shown in Fig. 8 (left). The capture criterion is obtained by substituting $\alpha=0$ and $R_{i}(\alpha)=R_{\text {tip }}$ in the above equation. The solution with the minus sign gives $d_{J C}^{-}=d_{J T}$ (see Eq. (4)), resulting in an infinite velocity ratio $V R_{\text {capt }}^{-}=\infty$. This solution represents the extreme operating condition where the slicing of the first scoop and capturing of the next scoop coincide. This case is further ignored, so that the capture criterion becomes

$$
V R_{\text {capt }}^{+}=\frac{\left[\cos ^{-1}\left\{\frac{d_{J C}^{+} \sin \left(\phi_{j e t}\right)}{R_{t i p}}\right\}-\cos ^{-1}\left\{\frac{d_{J T} \sin \left(\phi_{j e t}\right)}{R_{t i p}}\right\}+\frac{2 \pi}{N_{\text {scoops }}}\right] R_{t i p}}{d_{J C}^{+}-d_{J T}}
$$

The above result is the capture criterion, written in terms of a second critical velocity ratio. This dimensionless number is also a function of the jet angle, since $\theta_{1}, \theta_{2}=f\left(\phi_{\text {jet }}\right)$. If the velocity ratio is lower than the capture criterion, the jet is not fully captured (Fig. 8: right), which reduces the capture efficiency. An analytical expression for the capture efficiency in this low-speed region $\left(0<\omega<\omega_{c r}\right)$ is given in [10].

An example of capture conditions and the capture criterion is given in Fig. 9.

The range of jet angles valid for the capture conditions differs from that for the splashing conditions. To obtain this range we consider the angle between the two solutions, which is described by (Fig. 7: left and right)

$$
\cos \left(\frac{\theta_{1}-\theta_{2}}{2}\right)=\frac{R_{\text {jet }}}{R_{i}(\alpha)} \sin \left(\phi_{j e t}\right),
$$

or

$$
\phi_{\text {jet }}=\sin ^{-1}\left[\frac{R_{i}(\alpha)}{R_{\text {jet }}} \cos \left(\frac{\theta_{1}-\theta_{2}}{2}\right)\right] .
$$

Note that it is assumed here that the jet is captured by the next scoop. In that case the maximum angle is $\left(\theta_{1}-\theta_{2}\right)=2 \pi / N_{\text {scoops }}$. In principle the jet can be captured by the second next or even third next scoop. However, these cases are not considered, since it is usually prevented by the presence of the first scoop, inner shaft or other equipment. The minimum angle is $\left(\theta_{1}-\theta_{2}\right)=0$, in which case the jet touches, but never enters the circle with scoop tip radius. The minimum and maximum jet angles now follow from Eq. (26), which gives the range of jet angles

$$
\sin ^{-1}\left(\frac{R_{i}(\alpha)}{R_{\text {jet }}} \cos \left(\pi / N_{\text {scoops }}\right)\right)<\phi_{\text {jet }}<\sin ^{-1}\left(\frac{R_{i}(\alpha)}{R_{\text {jet }}}\right) .
$$

Note: The formulation of the capture conditions looks similar to that of the splashing conditions in Section 2. However, there are essential differences. The splashing conditions apply to the outer contour of a scoop. The capture conditions apply to the inner contour of the next scoop (represented by the term $2 \pi / N_{\text {scoops }}$ ), and here are two solutions. Consequently the range of operating conditions and shape of the splashing and capture curves are very different, as shown in the Figs. 5 and 9.

In Section 6 the capture criterion and capture conditions are applied to some scoop configurations.

\section{Experiments}

\subsection{Set-up}

Laboratory experiments were conducted using a scoop test module mounted onto a single shaft test facility at the G2TRC of the University of Nottingham. A schematic diagram of the facility with the test module is shown in Fig. 10. For further details refer to [10]. 


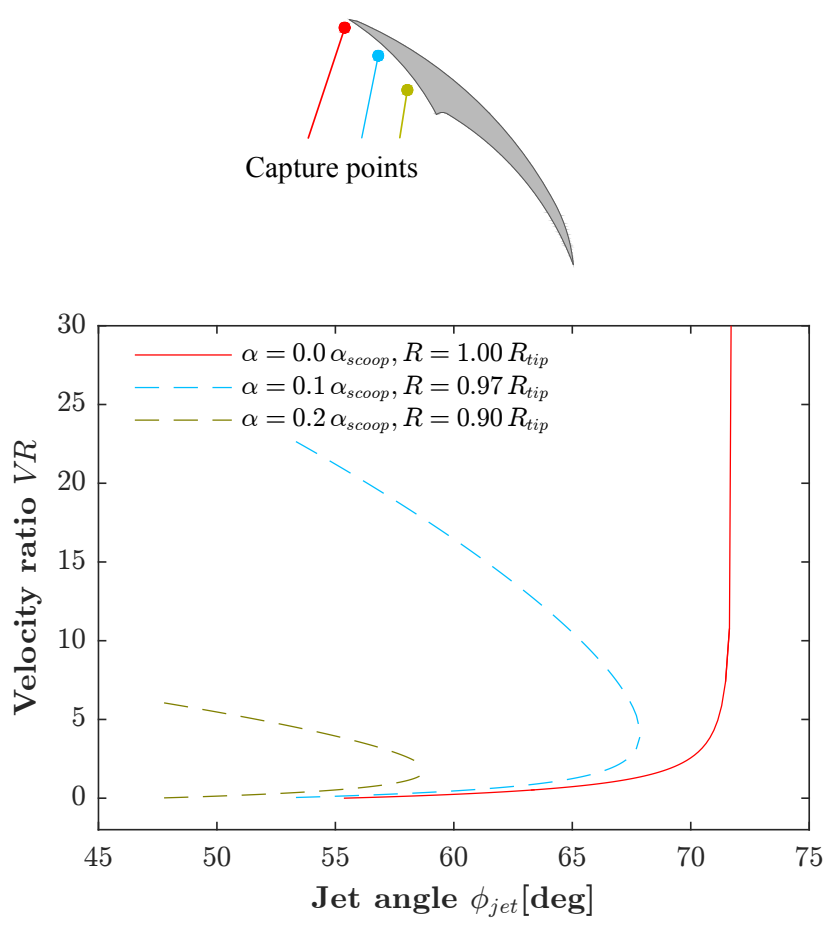

Figure 9: Scoop operating characteristic. Capture criterion (red) with capture point on scoop tip. Capture conditions (dashed lines) with capture points on inner scoop contour.

The scoop test module consists of two chambers: the front chamber and the scoop chamber, containing the scoops and oil injector, as shown in the photos in Fig. 11. Oil captured by each of the scoops is directed axially along the shaft through passages and collected in the front chamber. Each chamber has its own scavenge port, so that oil passing through the scoops is separated from oil not captured by the system.

A positive displacement pump delivers oil to the oil jet manifold at the required flow rate $Q_{j e t}$. The flow rate was adjusted by a pump inverter and measured using an Kral flowmeter (OME24, range up to $50 \mathrm{lpm}$ ). For these experiments Aeroshell 390 turbine oil was used, with the steady state feed temperature $T_{\text {jet }}$ kept within $30 \pm 5^{\circ} \mathrm{C}$. The density of the oil is $933.4 \mathrm{~kg} \mathrm{~m}^{-3}$, the kinematic viscosity is $2.71 \times 10^{-5} \mathrm{~m}^{2} \mathrm{~s}^{-1}$, and the oil/air surface tension coefficient is $3.12 \times 10^{-2} \mathrm{~N} \mathrm{~m}^{-1}$ (all values at $\left.30{ }^{\circ} \mathrm{C}\right)$.

The front chamber contains an exit port at the bottom through which oil exits under the effect of gravity via a flexible pipe into a container standing on electronic scales (Fig. 12). The mass of oil on the scales is logged during testing and used to calculate the captured oil flowrate. The oil flow rate from the front chambers was measured at each operating point. A positive displacement pump was used to empty the container after each test returning the oil to the oil reservoir.

The scoop chamber houses the rotating scoop element and the housing contains an exit port that provides gravity drainage for the oil not passing through the scoops. Lubrication oil for the rig front bearing also exits here. Oil leaving through the scoop chamber housing is collected in a tray and pumped back

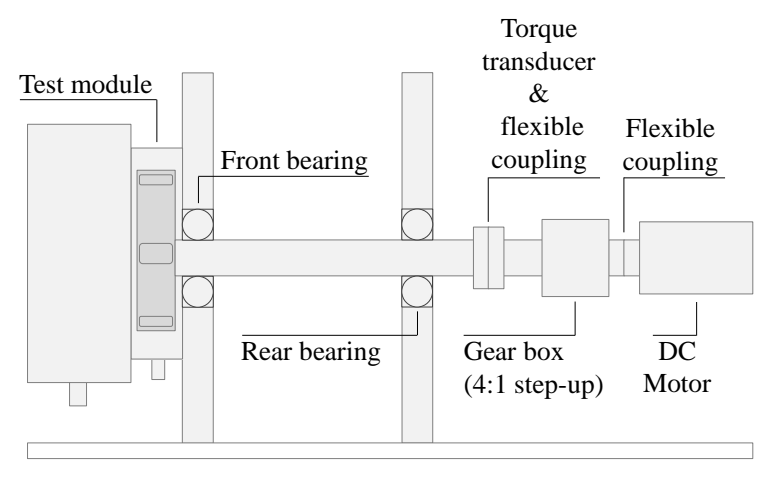

Figure 10: Schematic diagram of single shaft test facility with test module.

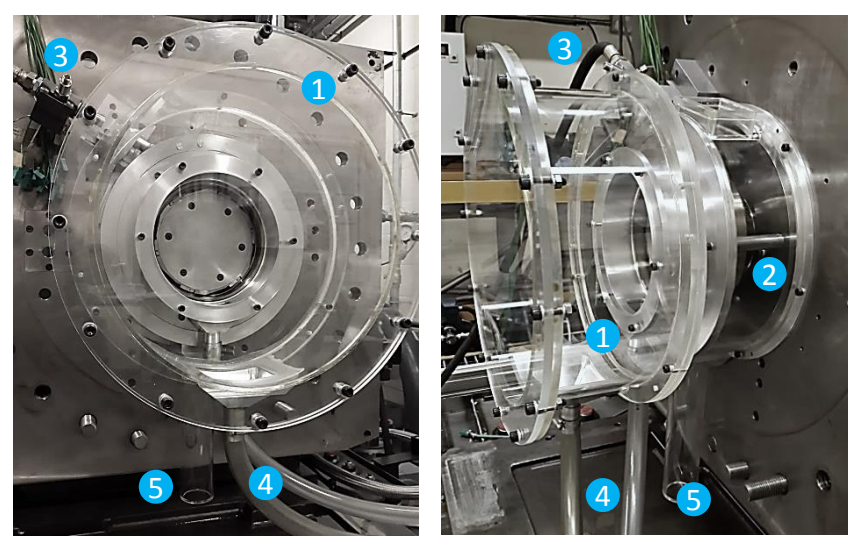

Figure 11: Oil scoop test facility. Front view (left) and side view (right) with components: 1-Front chamber, 2-Scoop chamber, 3- Oil jet injector, 4-Front chamber exit and 5-Scoop chamber drain.

to the oil reservoir. The top of the scoop chamber contains an opening for access of the borescope for flow visualization.

\subsection{Oil scoop capture efficiency}

The oil scoop capture efficiency is measured as the ratio of the oil mass flow captured and retained by the scoop $\left(\dot{M}_{\text {scoop }}\right)$ to the average of the oil mass flow injected to the scoop $\left(\dot{\bar{M}}_{\text {jet }}\right)$, that is

$$
\text { Efficiency }[\%]=\frac{\dot{M}_{\text {scoop }}\left[\mathrm{kg} \mathrm{s}^{-1}\right]}{\overline{\dot{M}}_{\text {jet }}\left[\mathrm{kg} \mathrm{s}^{-1}\right]},
$$

The duration of a capture efficiency test was typically between 5 and 8 minutes. The uncertainty in the capture efficiency is within $1.6 \%$ [10].

\subsection{Visualization}

To get a detailed insight in capture efficiency losses, the oil jet behaviour in the vicinity of the leading edge of the scoop was visualized.

The set-up for the visualization is shown in Fig. 13. A high speed camera (manufacturer IDT, model OS4-S3-M-04) was used. To improve the resolution and quality of images inside the scoop chamber a rigid borescope (Olympus series 5 zoom 


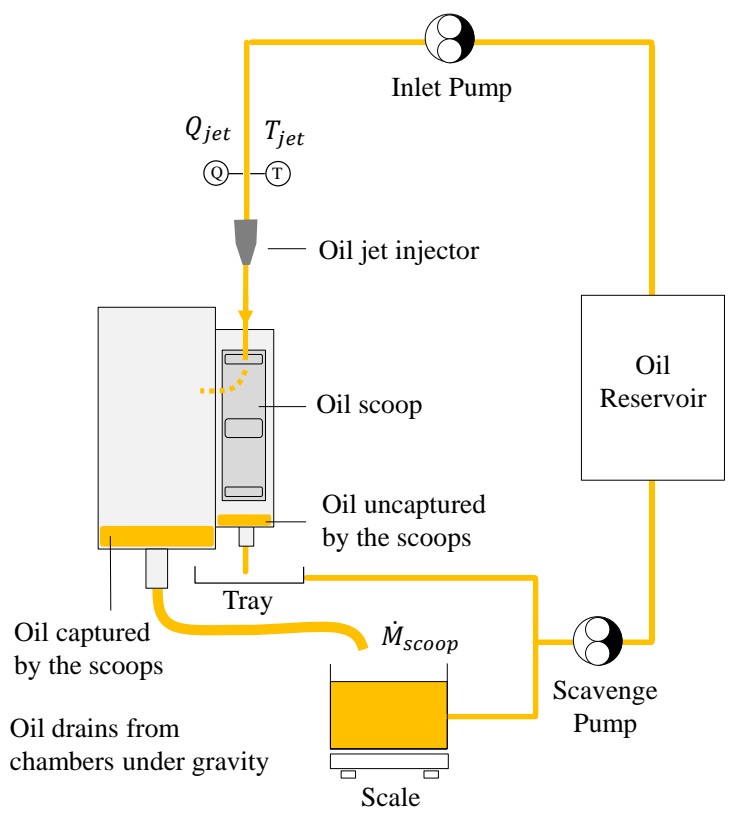

Figure 12: Schematic diagram of the oil scoop module and associated hydraulic circuit.

swing prism) was attached to the camera. For the illumination four white lights (Model 120E Veritas) were used. They were synchronized and pulsed with the high speed camera. Each light offered 22000 lumens output when pulsed.

\subsection{Test results}

Observation of the oil jet interaction with the scoop leading edge enables a good insight of the scoop efficiency losses. Visualisation tests are performed at relatively low shaft speeds and low jet flow rates to avoid that the borescope lens is covered with splashed oil, making the image blurred. The main observed features reveal different forms of efficiency losses:

- Oil that is not captured by the scoops. The slicing of the jet by a scoop edge results in the formation of plumes, ligaments and droplets. Due to their outward motion, these are not captured by the next scoop. In Fig. 14 (top), the deflected plume is observed along with ligaments and droplets.

- Oil that is initially captured by a scoop, but is lost at a later stage. Due to centrifugal losses oil is accelerated outwards and leaves the scoop. In Fig. 14 (bottom) centrifugal losses are shown. It is interesting to see that in this case they appear in the form of regularly distributed oil lines, arising from the scoop edge.

- Oil that may or may not be captured at a later stage. After jet slicing some oil remains attached to the outer scoop surface, forming a thin film. Due to the combination of centrifugal losses and surface tension, droplets are formed from this film. Some of these droplets will be captured after release from the outer surface.

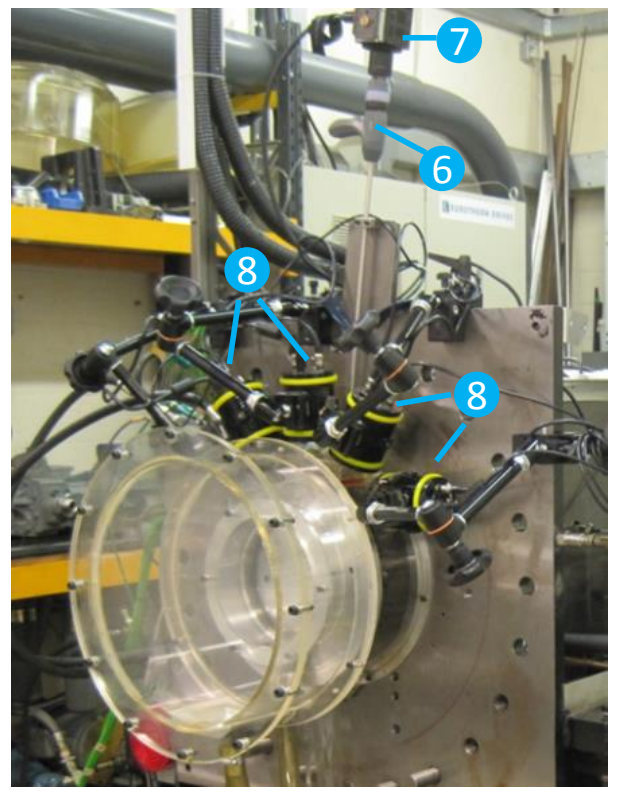

Figure 13: Visualization set up. 6-Borescope, 7-Camera and 8-Lights.
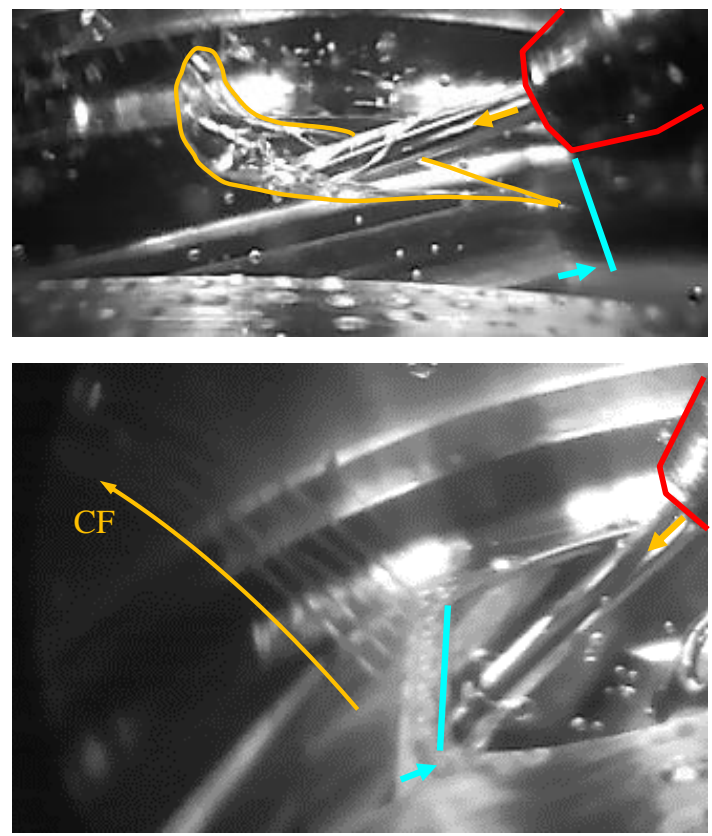

Figure 14: Visualisation test. Oil jet injector (red), jet direction (yellow arrow). Top: Formation of plume (contour marked with a yellow line), ligaments and droplets after jet slicing by scoop edge (blue). Bottom: Centrifugal losses (CF) in the form of oil jets leaving via scoop edge.

Observed phenomena around the plume formation are:

- The size of the plume is smaller at small jet angles. In those cases, the jet is sliced in a plane more perpendicular to its axis, reducing the cross sectional sliced area. This trend is seen in experiments performed at constant $V R$.

- The more the jet is directed parallel to the scoop contour, the more the inner side of the plume is suppressed. At high jet angles the plume appears at the outer side only, while at 
low jet angles, the plume appears at both sides in the form of a "horse shoe". The single sided plume grows faster and stretches more than the double sided plume.

The capture efficiency tests are performed up to shaft speeds of $10000 \mathrm{rpm}$ and jet angles between $25^{\circ}$ and $65^{\circ}$. At each jet angle, the velocity ratio is increased from about 1 to 10 with stepwise increments of 1 . The velocity ratio is adjusted in two ways: 1) in one series the jet velocity is varied at a constant shaft speed $(\omega=5000 \mathrm{rpm})$, and 2$)$ in the other series the shaft speed is varied at a constant jet velocity $\left(V_{j e t}=12 \mathrm{~m} \mathrm{~s}^{-1}\right)$. The test results at a jet angle $\phi_{j e t}=30.0^{\circ}$ are shown in Fig. 15. The efficiency characteristics at constant shaft speed and constant jet velocity are about similar for low velocity ratios up to 5 . The differences at higher velocity ratios are more profound. The results at a jet angle $\phi_{\text {jet }}=64.2^{\circ}$ are shown in Fig. 16. Here the two efficiency characteristics show smaller differences over the entire range of velocity ratios. The efficiency strongly increases with decreasing velocity ratio with a maximum value at $V R=1$.

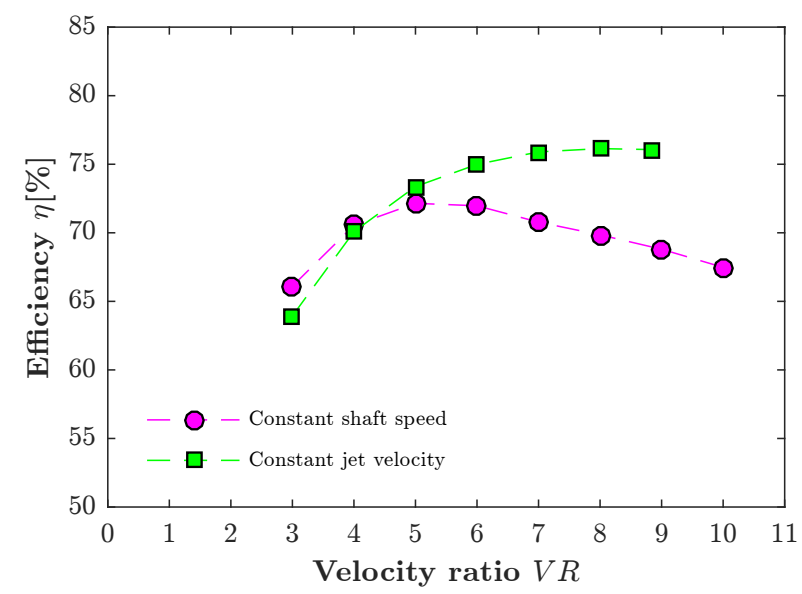

Figure 15: Capture efficiency characteristic $\left(\phi_{\text {jet }}=30.0^{\circ}\right)$

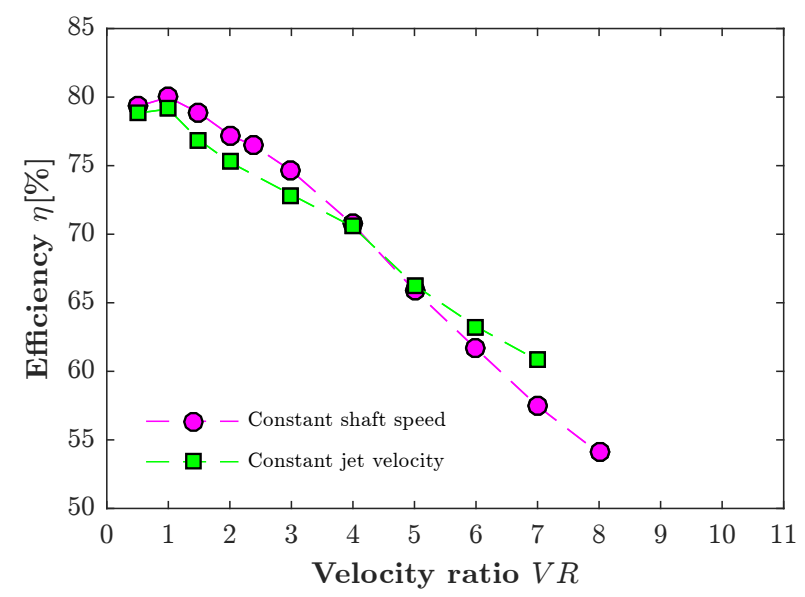

Figure 16: Capture efficiency characteristic $\left(\phi_{\text {jet }}=64.2^{\circ}\right)$

To explore the differences in capture efficiency between the two test series, the jet velocity relative to the scoop tip velocity is considered. The radial $\left(V_{r}\right)$ and tangential $\left(V_{t}\right)$ components of the relative jet velocity are

$$
\begin{aligned}
& V_{r}=V_{j e t} \cos \phi_{j e t} \\
& V_{t}=V_{j e t} \sin \phi_{j e t}+V_{t i p} .
\end{aligned}
$$

Between the two test series, the differences in the efficiency, radial and tangential velocities, are evaluated at the same velocity ratio as

$$
\begin{aligned}
\Delta \eta(V R) & =\eta\left(V_{\text {jet }}=12 \mathrm{~m} \mathrm{~s}^{-1}, V R\right)-\eta(\omega=5000 \mathrm{rpm}, V R) \\
\Delta V_{r}(V R) & =V_{r}\left(V_{\text {jet }}=12 \mathrm{~m} \mathrm{~s}^{-1}, V R\right)-V_{r}(\omega=5000 \mathrm{rpm}, V R) \\
\Delta V_{t}(V R) & =V_{t}\left(V_{\text {jet }}=12 \mathrm{~m} \mathrm{~s}^{-1}, V R\right)-V_{t}(\omega=5000 \mathrm{rpm}, V R) .
\end{aligned}
$$

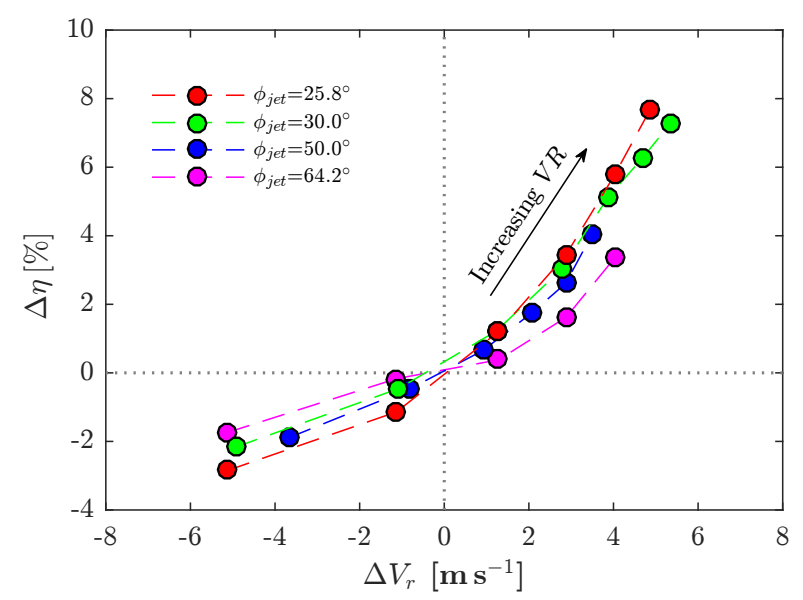

Figure 17: Comparison of two test series at constant shaft speed and constant jet velocity. Difference in capture efficiency as function of the difference in radial component of the relative jet velocity.

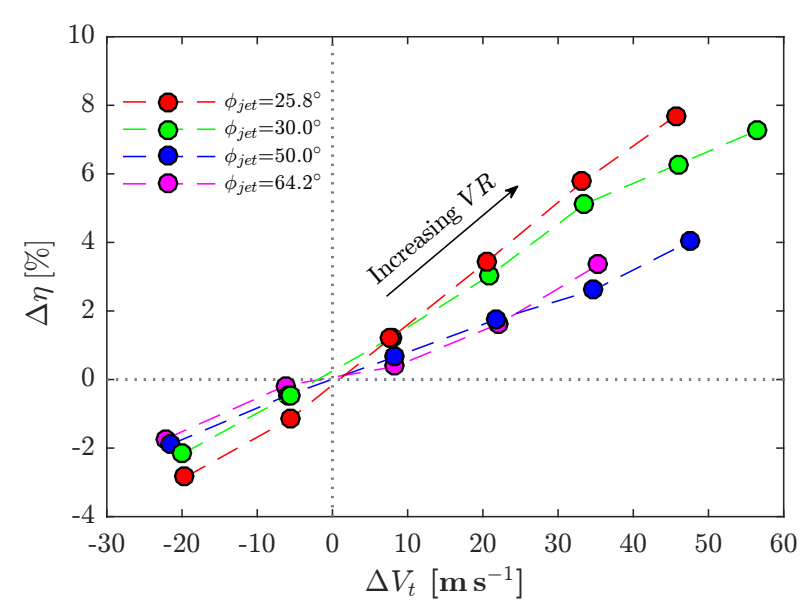

Figure 18: Comparison of two test series at constant shaft speed and constant jet velocity. Difference in capture efficiency as function of the difference in tangential component of the relative jet velocity. 
In Fig. 17 the difference in capture efficiency $(\Delta \eta)$ is given as function of the difference in radial component $\left(\Delta V_{r}\right)$ of the relative jet velocity. In Fig. 18 this difference is given as function of the tangential component $\left(\Delta V_{t}\right)$. Note that at the cross point of the two test series the velocity ratio $V R=$ 4.4. At this cross point the capture efficiencies and relative jet velocities are the same, so that their differences are zero. From these figures it may be concluded that the capture efficiency increases with increasing relative jet velocity. The increase of the efficiency with the radial component is about the same for all jet angles (Fig. 17). The increase of the efficiency with the tangential component is higher at lower jet angles and about linear for all jet angles (Fig. 18). It may be expected that the oil jet becomes deeper inserted into the scoop, when the tangential component is higher. This on its turn reduces the oil losses due to centrifugal forces. The increase of the efficiency with the relative jet velocity, at the same velocity ratio, may therefore (at least partially) be attributed to a reduction of the centrifugal losses.

All test results at a constant shaft speed $(\omega=5000 \mathrm{rpm})$ are presented in Fig. 19, where the capture efficiency characteristic is presented as a function of the jet angle and velocity ratio. It can be observed that the peak value of the efficiency is less pronounced at low jet angles, while at higher jet angles the peak value is sharp and well defined. In order to fill the entire domain of operating conditions, the test points are interpolated to intermediate jet angles $\Delta \phi_{\text {jet }}=5^{\circ}$ and velocity ratios $\Delta V R=1$. The projection of the capture efficiency characteristic in Fig. 20 provides contours of constant efficiency. These graphs show that the peak values of the efficiency move from high velocity ratios at low jet angles to low velocity ratios at high jet angles. The tendency of these peak values, as represented by the best efficiency curve, is further discussed in Section 5. All test results at a constant jet velocity $\left(v_{\text {jet }}=12 \mathrm{~m} \mathrm{~s}^{-1}\right)$ are presented in Figs. 21 and 22. The trends shown here are very similar to those at constant shaft speed (Figs. 19 and 20).

\section{Validation and best efficiency characteristics}

In this section the basic assumption of this paper, i.e. the best scoop efficiency is correlated to the splashing criterion, is validated against experiments.

The splashing criterion (Section 2) and capture criterion (Section 3) are applied to the tested scoop configuration and compared with the experimental data. The peak values of the experimentally obtained efficiency are interpolated from the efficiency curves at the four jet angles. This is done for both test series at constant shaft speed (see Fig. 19) and constant jet velocity (see Fig. 21), resulting in 8 points of best efficiency.

In Fig. 23 the analytical splashing and capture criteria are plotted together with the 8 operating points of best efficiency. The splashing criterion (blue line) represents the operating conditions, at which the oil jet is aimed to hit the rear side of the scoop. The effect of the jet diameter (i.e. $0.002 \mathrm{~m}$ ) on the splashing criterion is taken into account (see Applications
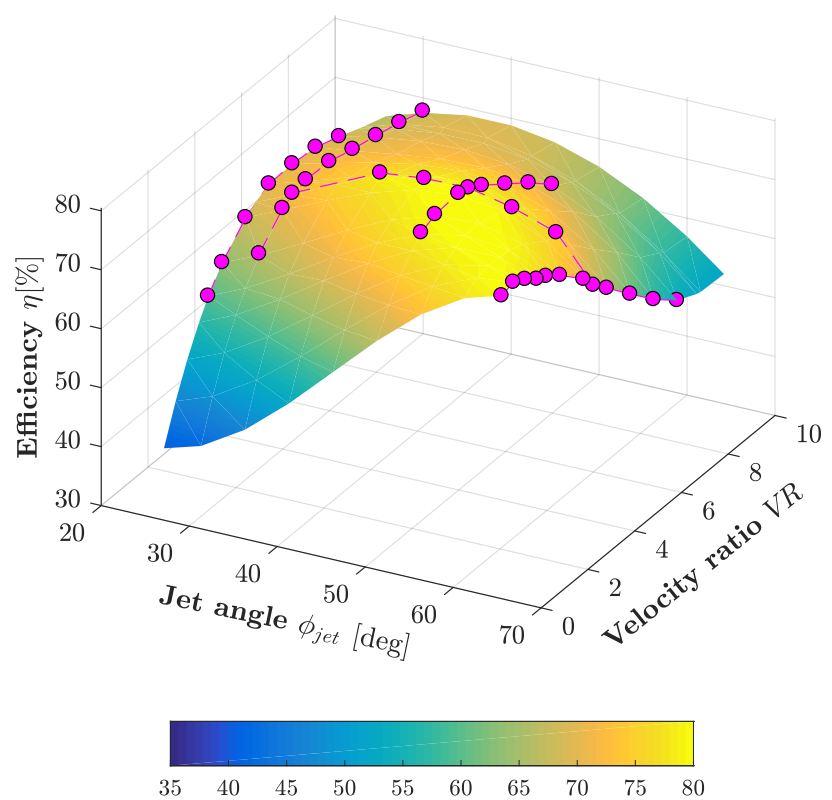

Figure 19: Capture efficiency characteristic. Experimental data at constant shaft speed (circles) with interpolated values (colour bar).

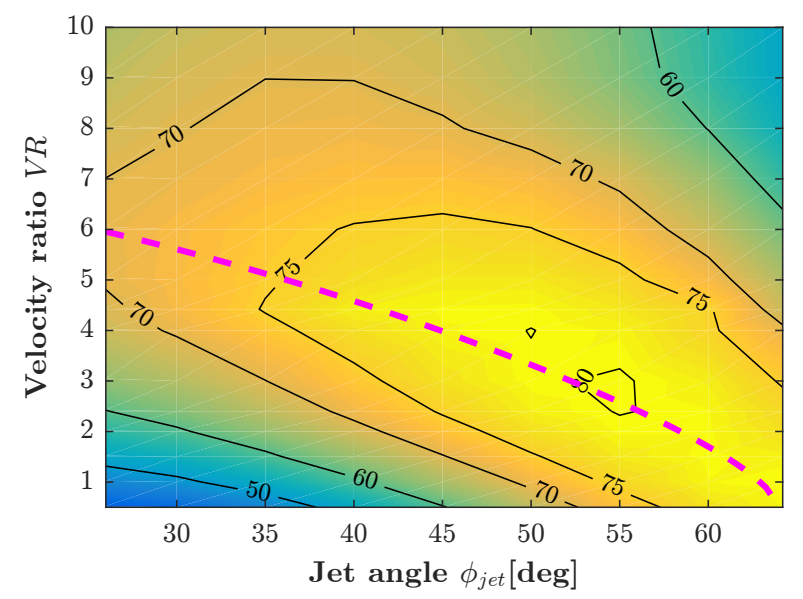

Figure 20: Capture efficiency characteristic. Contour plot with interpolated values from experimental data at constant shaft speed. Best efficiency curve (dashed line) is obtained from analytical method

in Section 6.1). Velocity ratios below the splashing criterion result in splashing on the outer scoop contour. The dashed lines represent fixed splashing points at $5^{\circ}$ (green) and $19^{\circ}$ (magenta) from the rear side of the scoop. These dashed lines match well with the experimental data and as such represent curves of best efficiency. The area between the dashed lines (grey) thus represents the operating conditions of best efficiency. The capture criterion (red line) represents the operating conditions, at which the oil jet is aimed to hit the scoop tip. At velocity ratios above the capture criterion the jet is fully captured by the scoops. At velocity ratios below the capture criterion part of the jet is missed, which results in a loss of efficiency. However, at these low velocity ratios scoops may be used to control the 


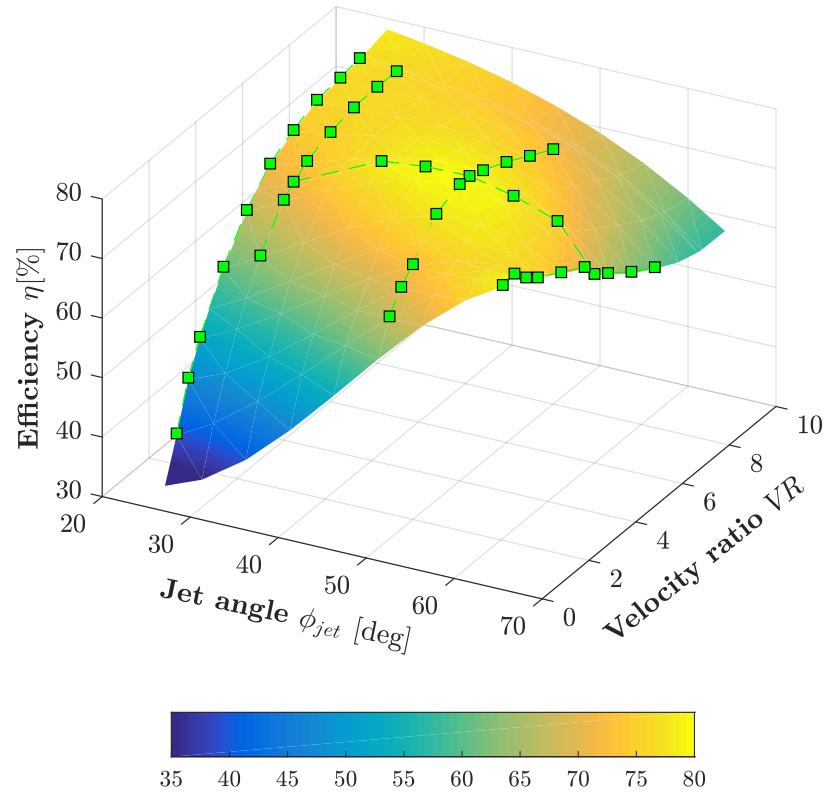

Figure 21: Capture efficiency characteristic. Experimental data at constant jet velocity (squares) with interpolated values (colour bar).

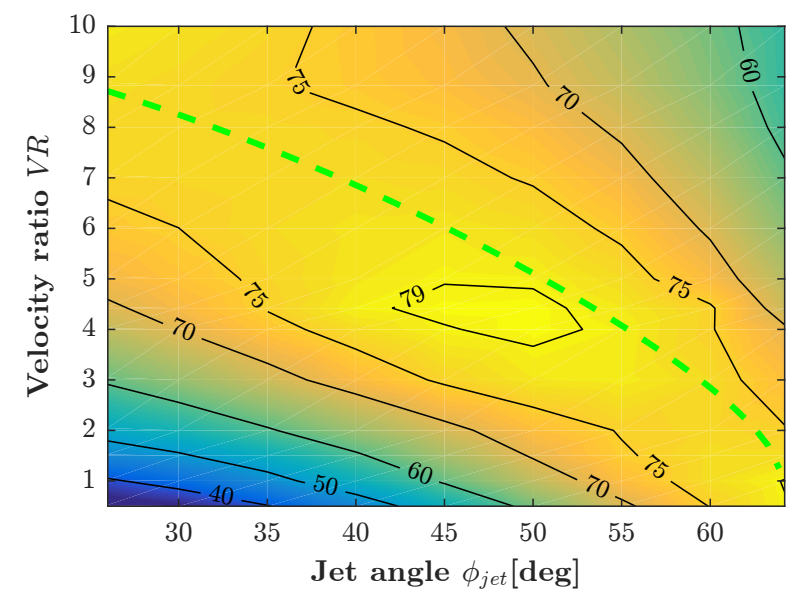

Figure 22: Capture efficiency characteristic. Contour plot with interpolated values from experimental data at constant jet velocity. Best efficiency curve (dashed line) is obtained from analytical method.

oil distribution, i.e. how much oil is directed into the scoops and how much oil is used for lubrication elsewhere.

From the above the following conclusions may be drawn: The curves of best efficiency represent fixed splashing points on the outer scoop contour, located near the rear side of the scoop. This holds for any jet angle, although the experimental data show that the efficiency varies along the best efficiency curves. The curves are obtained from splashing conditions and as such they are correlated to the splashing criterion, representing splashing on the rear side. It is this correlation that allows for an estimation of the range of operating conditions of best efficiency. By defining the basic dimensions (i.e. scoop tip and rear radius and jet angle and position, ) the splashing criterion can be determined from this analytical approach. If the band of fixed splashing points of best efficiency is known, obtained from scaling, or otherwise estimated for a scoop design, the range of operating conditions of best efficiency can be calculated. This approach allows for the specification of the operating conditions at the design stage, to achieve the best efficiency, although it does not provide the magnitude of the best capture efficiency.
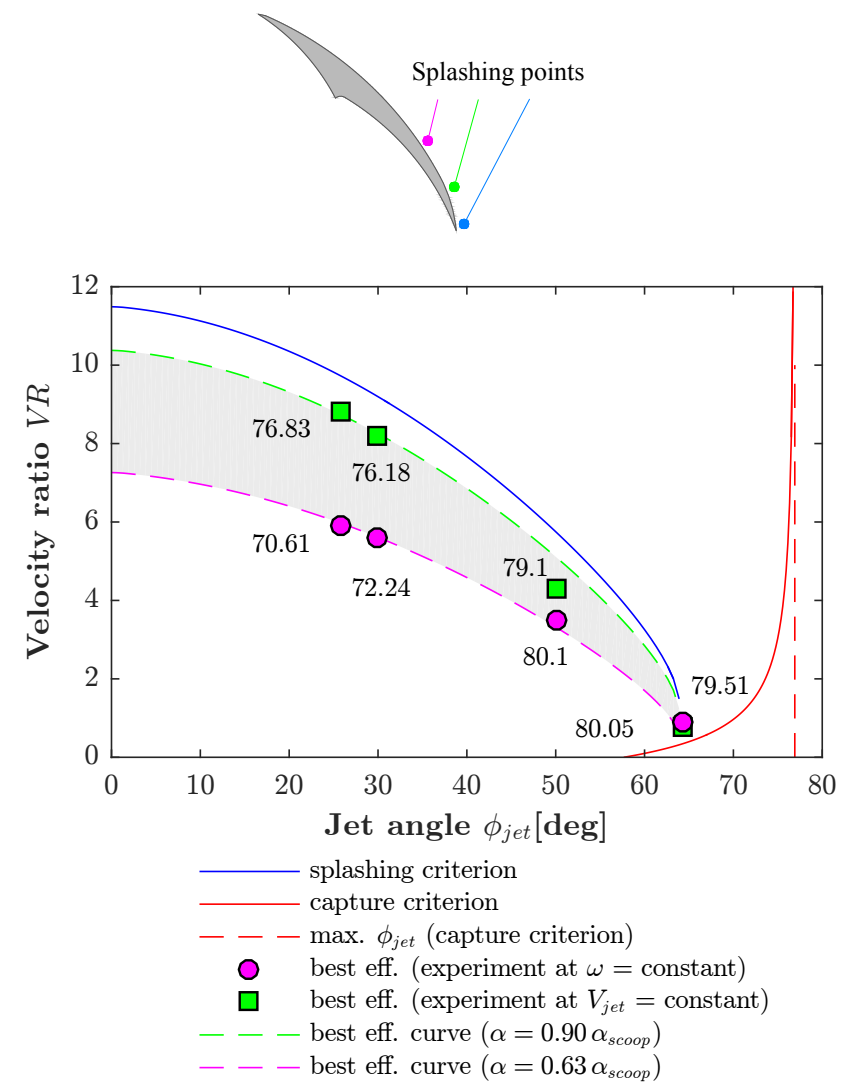

Figure 23: Scoop operating characteristic. Range of operating conditions for best efficiency (grey). No splashing, above the splashing criterion (blue). No full capture, below the capture criterion (red).

The increase of the capture efficiency under splashing conditions may be attributed to the following combination of phenomena:

- The contraction of "horse shoe" plumes, which is followed by a reattachment to the jet, so that a larger part of the plume is captured. The "horse shoe" plumes are seen in experiments at lower jet angles (see Section 4.4: plume formation). The contraction is seen in experiments at low shaft and jet speeds (Fig. 24) and may be attributed to the drag force from the surrounding air flow.

- The splashing of the plume on the outer scoop contour. A mixed oil-air film is formed, which is partially captured by the next scoop. The splashing of the plume is seen in experiments at high shaft and jet speeds (Fig. 25). 
- The jet angle under splashing conditions is lower than that under no splashing conditions, at the same velocity ratio (horizontal lines in Fig. 23). At lower jet angles the plume tends to be more double sided and less stretched (Section 4.4: plume formation). A larger part of the slightly rotated and smaller plume is more likely to be captured.

- In case of plume formation, the jet front will no longer grow linear in time, but slower. Although the jet is aimed to hit the outer rear side of a scoop, it may no longer splash, and is fully captured.

- The splashing of the jet on the outer scoop contour results in the formation of droplets. As a secondary effect, part of the bouncing droplets are captured by the next scoop. Droplets are always seen in experiments.
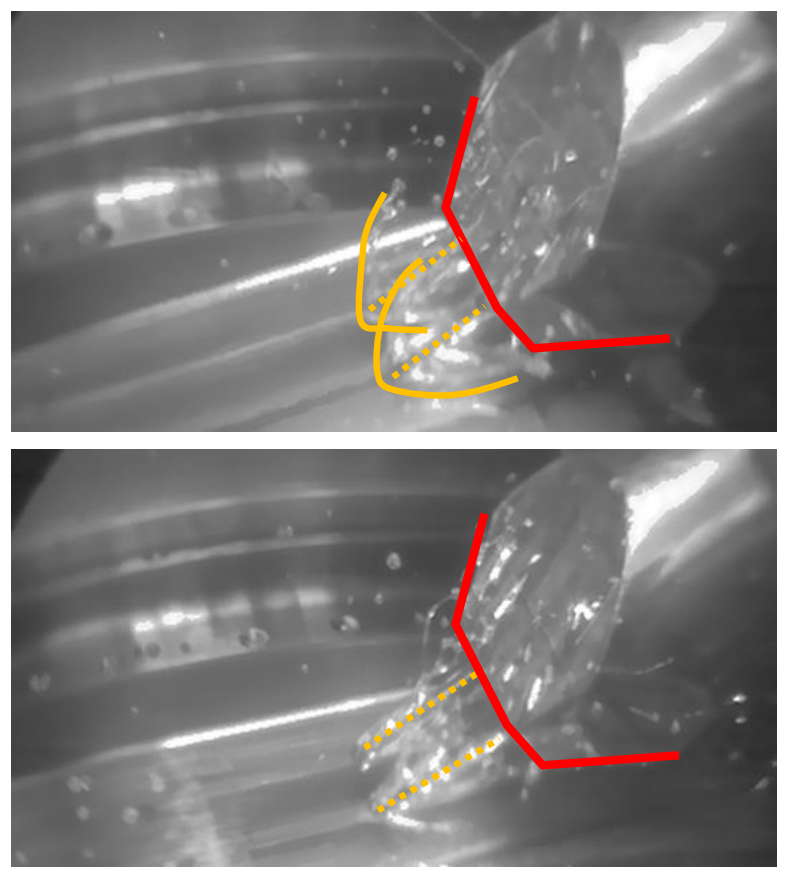

Figure 24: Contraction of plumes. Oil jet injector (red). Top: "horse shoe" plumes (contour marked with yellow line) at low shaft and jet speeds ( $V R=$ 4.4). Bottom: Contraction of plumes.

\section{Applications}

In this section the analytical approach is utilized to illustrate how it may be used in scoop design. For this purpose, the splashing and capture criteria are applied to fictive scoop configurations. The splashing criterion in Eq. (12) describes the set of operating conditions between splashing and no splashing on the outer scoop contour. For velocity ratios higher than the splashing criterion no splashing takes place. The capture criterion in Eq. (24) describes the set of operating conditions between a full and partial jet capture. For velocity ratios higher than the capture criterion, the jet is fully captured
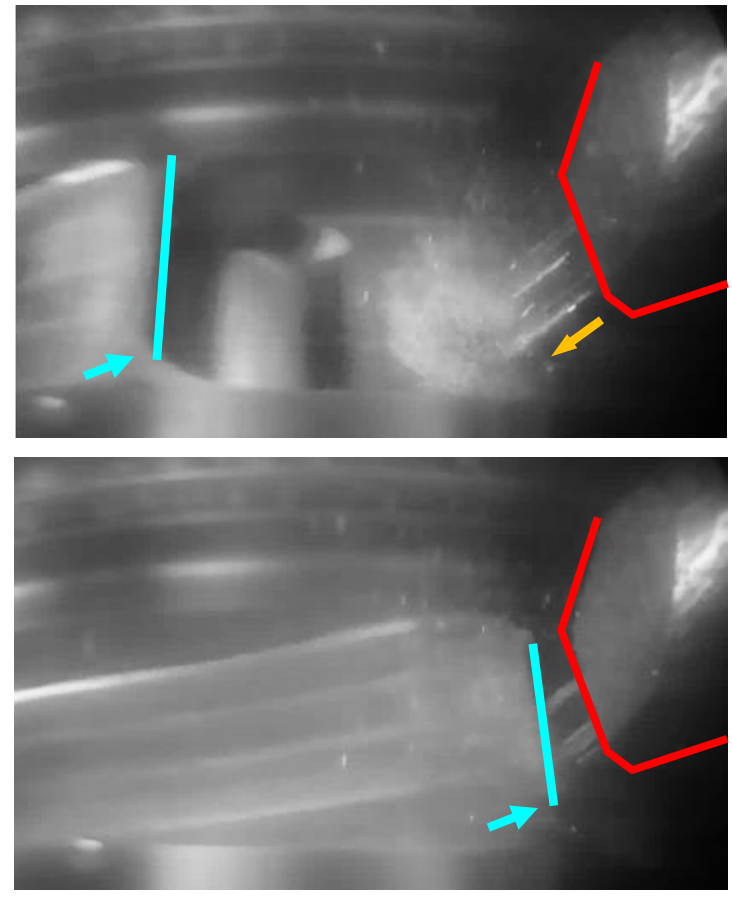

Figure 25: Splashing of plumes. Oil jet injector (red), jet direction (yellow arrow) and scoop edge (blue). Top: Splashing of the jet at relatively high shaft and jet speeds $(V R=4.4)$. Bottom: The mixed oil-air film formed is partially captured by the scoop.

by the scoop.

The fictive scoop configuration consists of 6 scoops with the following specifications (unless otherwise specified)

$$
\frac{R_{\text {tip }}}{R_{\text {jet }}}=0.95, \quad \frac{R_{\text {rear }}}{R_{\text {tip }}}=0.90, \quad \alpha_{\text {scoop }}=50^{\circ} .
$$

\subsection{Example 1: Effect of jet diameter}

In the first example the effect of the jet diameter is shown. This effect is taken into account as a change of the radial jet position (see Fig. 26) according to

$$
\Delta R_{j e t}=\frac{d_{j e t} / 2}{\left|\sin \left(\phi_{j e t}\right)\right|} .
$$

The effect of the jet diameter on the splashing and capture criteria is shown in Fig. 27. The three curves represent the jet centre line (as if the jet diameter is zero), and the inner and outer sides of the jet. As a result the splashing criterion at the inner jet side is the most critical.

\subsection{Example 2: Effect of conical jet or plume}

In the second example the effect of a conical jet or plume on the splashing criterion is considered. In both cases the effect is taken into account as an increment of the jet angle from the jet centre line

$$
\Delta \phi_{\text {jet }}= \pm \theta_{\text {cone }} / 2 \quad \text { or } \quad \Delta \phi_{\text {jet }}= \pm \theta_{\text {plume }} / 2
$$




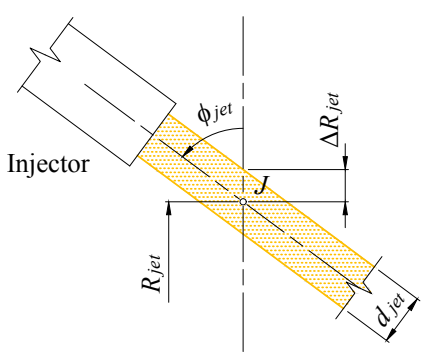

Figure 26: Radial positions of inner and outer jet.

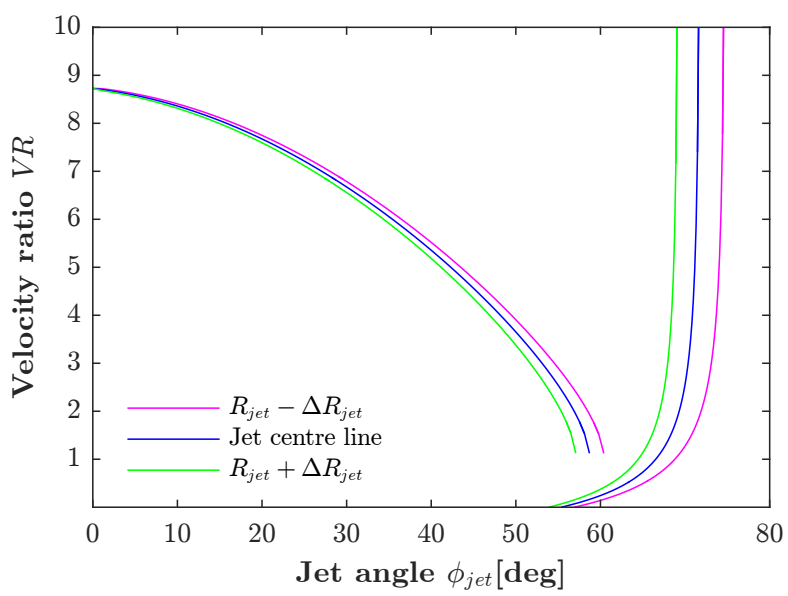

Figure 27: Example 1. Effect of jet diameter on splashing criterion (left) and capture criterion (right). The curves represent the inner jet (magenta), jet centre line (blue) and outer jet (green). $\Delta R_{\text {jet }}=0.001 \mathrm{~m}$.

The effect on the splashing and capture criteria is shown in Fig. 28. This effect is similar to that in the first example. Also here the splashing criterion is defined by the inner jet. The cone angle depends on the type of injector. The plume angle is less well defined. It depends on the operating conditions, and due to the scoop slicing, may be different at either side of the jet. The splashing criterion at the inner jet side is again the most critical.

\subsection{Example 3: Effect of number of scoops}

In the third example the effect of the number of scoops is considered. This effect is taken into account as a change in the scoop angle according to

$$
\alpha_{\text {scoop }}=\frac{300}{N_{\text {scoops }}} ; \quad \alpha_{\text {gap }}=\frac{60}{N_{\text {scoops }}} ; \quad \frac{R_{o}\left(\alpha / \alpha_{\text {scoop }}\right)}{R_{\text {tip }}},
$$

where $\alpha_{\text {gap }}=360 / N_{\text {scoops }}-\alpha_{\text {scoop }}$, so that the ratio $\alpha_{\text {gap }} / \alpha_{\text {scoop }}$ remains constant. Geometrical similarity of the "stretched" scoops is satisfied by the third dimensionless group. The effect on the splashing and capture criteria is shown in Fig. 29. The critical velocity ratio of the splashing criterion increases with a decreasing number of scoops, which implies that at high shaft speeds less scoops are needed. The capture criterion is hardly affected by the number of scoops, since it mainly depends on

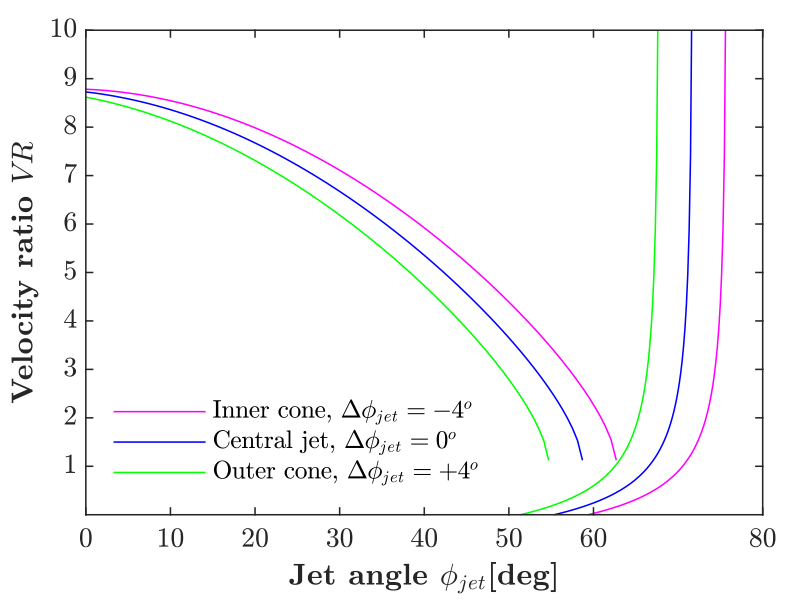

Figure 28: Example 2. Effect of conical jet or plume on splashing criterion (left) and capture criterion (right). The curves represent the outer jet (green), jet centre line (blue) and inner jet (magenta), for $\theta_{\text {cone }}=8^{\circ}$.

the ratio $R_{t i p} / R_{\text {jet }}$, which is kept constant here.

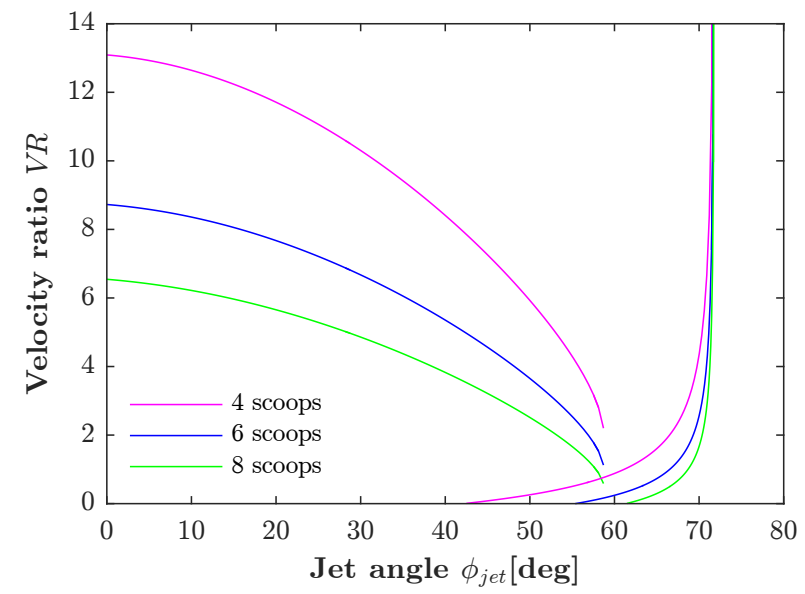

Figure 29: Example 3. Effect of number of scoops on splashing criterion (left) and capture criterion (right).

\subsection{Example 4: Scaling to other scoop configurations}

In the fourth example we aim for the scaling of scoop configurations, such that they have the same splashing criterion. For this purpose two necessary conditions are imposed. The first condition is that the critical velocity ratio at a jet angle $\phi_{j e t}=0$ must be the same. The second condition is that the maximum (possible) jet angle (i.e. the asymptote in the splashing criterion) must be the same. The maximum jet angle is given in Eq. (8), when applied to the rear side of the scoop $\left(R_{o}(\alpha)=R_{\text {rear }}\right)$. From the above two conditions and Eq. (12), it follows that the following dimensionless parameter groups must be the same

$$
\gamma=\frac{R_{\text {tip }}-R_{\text {rear }}}{\alpha_{\text {scoop }} R_{\text {tip }}} \quad ; \quad \frac{R_{\text {rear }}}{R_{\text {jet }}} \quad ; \quad \frac{R_{o}\left(\alpha / \alpha_{\text {scoop }}\right)}{R_{\text {tip }}} .
$$


Note that the first parameter group may be seen as an angle of incidence $\gamma$, as if the scoop is an aerofoil, with the cord length in the denominator. The third group is added to describe the geometrical similarity of the "stretched" scoops. From the first and second dimensionless groups it follows that

$$
R_{\text {tip }}=\frac{R_{\text {rear }}}{1-\gamma \alpha_{\text {scoop }}} .
$$

In this example $\alpha_{\text {scoop }}, R_{\text {jet }}$ and $R_{\text {rear }}$ are kept the same as in the third example, so that only $R_{\text {tip }}$ changes according to the above equation. The results of three fictive scoop configurations are shown in Fig. 30. The three curves of the splashing criterion are practically the same. The asymptote of the capture criterion is not the same anymore, since the ratio $R_{t i p} / R_{\text {jet }}$ is no longer constant.

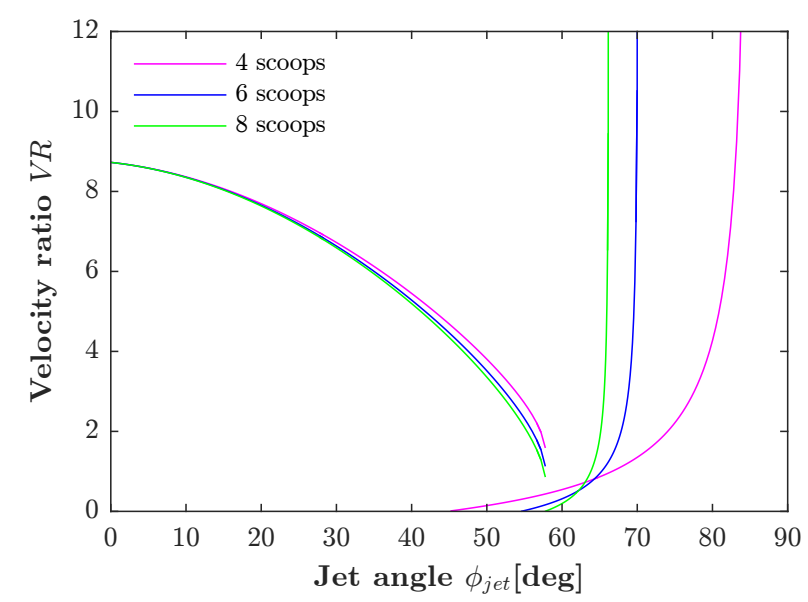

Figure 30: Example 4. Scaling of scoop configurations with the splashing criterion (left) and capture criterion (right).

\subsection{Example 5: Shaping of the scoop profile}

In this example the contour of the jet front relative to that of the rotating scoop is considered, as described in Section 2.1. Note that the equations in this section are valid for a specific jet angle.

For the original fictive scoop configuration with 6 scoops, the jet contours at two jet angles $\left(\phi_{j e t}=20^{\circ}\right.$ and $\left.63^{\circ}\right)$ are given in Fig. 31. The results show that the effect of the jet angle on the contour of the jet front is small. The jet contour becomes slightly less curved at high jet angles. It thus may be concluded that the highest jet angle is the most restrictive in the shaping of the scoop contour, such that it does not touch the oil jet.

The above examples demonstrate how the scoop configuration, described by the number of scoops and scoop dimensions, have an effect on the operating conditions, in terms of a velocity ratio and jet angle, and whether splashing and full capturing will occur or not.

In practice the shaft speed is dictated by the operating conditions of an aero-engine. The jet angle and/or number of scoops can be chosen to aim for low or high velocity ratios. Then, the velocity ratio can be controlled by choosing the jet velocity.

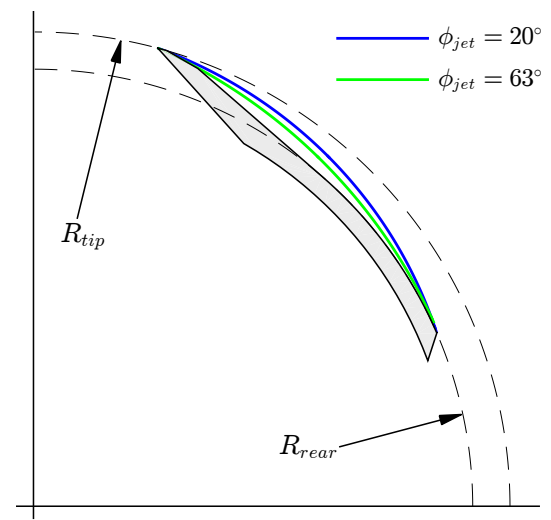

Figure 31: Example 5. The contour jet relative to the rotating scoop.

\section{Conclusions}

In this paper it is demonstrated that the best capture efficiency of a scoop configuration is obtained when the jet is aimed at the outer scoop contour near the rear side. An analytical method is introduced to describe the range of operating conditions at which splashing takes place. This approach differs from common practice, where the focus is solely on oil capture.

The operating conditions are represented in a scoop operating characteristic, where the splashing and capture criteria are presented as function of the dimensionless velocity ratio and the jet angle. The splashing criterion is used to define the operating conditions at which splashing on the outer surface takes place. While the capture criterion helps to ensure that the oil jet is not missed by the scoop at very low velocity ratios.

Experiments are performed at a range of velocity ratios and jet angles. The experimental data is used to validate the basic assumption in this paper, that the best scoop capture efficiency is correlated to the splashing criterion. It is shown that the trend of these best efficiency points can well be described by splashing conditions, at which the jet is aimed to hit a fixed point on the outer scoop contour near the rear side. This fixed point (of best efficiency) does not change with the jet angle, although the experiments have shown that the efficiency varies along the best efficiency curve.

The splashing and capture criteria are applied to fictive scoop configurations to demonstrate the effect of the jet diameter, conical jet or plume, number of scoops on the operating conditions. Scale laws are derived in terms of dimensionless parameter groups, for the scaling a scoop configuration to other geometrically similar configurations that share the same splashing criterion.

The analytical method may be useful in the scoop design to specify the operating conditions for the best efficiency. The splashing criterion can easily be obtained for a scoop configu- 
ration. If the main parameters (i.e. tip and rear scoop radius, jet angle and position) are known, the splashing and capture criteria can be calculated. For the best efficiency, velocity ratios below the splashing criterion and above the capture criterion should be chosen. If the range of fixed splashing points of best efficiency (near the rear side of the scoop) is known, obtained from scaling, or otherwise estimated, the range of operating conditions of best efficiency can be calculated. In practice the shaft speed is dictated by the operating conditions of an aero-engine. The jet angle and/or number of scoops can then be chosen to aim for low or high velocity ratios. And the velocity ratio can be controlled by choosing the jet velocity.

Further validation of this analytical method is needed. Experiments and CFD simulations should give insight in how far the correlation between the best efficiency characteristics and fixed splashing points on the outer contour also holds for other scoop configurations.

\section{References}

[1] E. V. Zaretsky, "Rolling Bearing and Gear Lubrication", 1997. Society of Tribologists and Lubrication Engineers, Park Ridge, Ill., US, pp. 207-323.

[2] S. I. Pinel, H. R. Signer, E. V. Zaretsky, "Comparison Between Oil-Mist and Oil-Jet Lubrication of High-Speed, Small-Bore, Angular-Contact Ball Bearings", Tribology Transactions, 44 (3), pp. 327-338 (2001). DOI:10.1080/10402000108982465.

[3] P. F. Brown, "Bearings and Dampers for Advanced Jet Engines", Technical Report, 1970. SAE Technical Paper No. 700318, Warrendale, Pennsylvania, US.

[4] C. W. Lee, G. R. Johnson, P. C. Palma, K. Simmons, S. J. Pickering, "Factors Affecting the Behaviour and Efficiency of a Targeted Jet Delivering Oil to a Bearing Lubrication System", Proceedings of ASME Turbo Expo 2004, Vienna, Austria, ASME Paper No. GT2004-53606, 2, pp.191-199.

[5] S. Kovaleski, "Radial Scoop Construction", 1987. US Patent 4,648,485.

[6] K. Fisher, H. Demel, R. Hazeley, "Methods and Apparatus for Supplying Oil to Bearing Assemblies", 2002. US Patent 6,409,464.

[7] K. Fisher, "Bi-Directional Oil Scoop for Bearing Lubrication", 2004. US Patent 6,682,222.

[8] J. Dins, J. Hogan, A. Kumar, "Curved Blade Oil Scoop", 2007. US Patent 7,244,096.

[9] S. McDonagh, "A Liquid-Capturing Shaft", 2016. EP Patent App. EP20,150,180,019.

[10] P. P. Cageao, K. Simmons, A. Prabhakar, B. Chandra, "Assessment of the Oil Scoop Capture Efficiency in High Speed Rotors", Journal of Engineering for Gas Turbines and Power, ASME GTP-17-1167 (in press, 2017).

[11] G. Tryggvason, M. Ma, J. Lu, "DNS - Assisted Modeling of Bubbly Flows in Vertical Channels", Nuclear Science and Engineering, 184 (3), pp. 312-320 (2016). DOI:10.13182/NSE16-10.

[12] M. Ma, J. Lu, G. Tryggvason, "Using Statistical Llearning to Close TwoFluid Multiphase Flow Equations for a Simple Bubbly Sytem", Physics of Fluids, 27092101 (2015). DOI: 10.1063/1.4930004.

[13] M. Ma, J. Lu, G. Tryggvason, "Using Statistical Llearning to Close TwoFluid Multiphase Flow Equations for Bubbly Flows in Vertical Channels", International Journal of Multiphase Flow, 85 (3), pp. 336 - 347 (2016). DOI: 10.1016/j.ijmultiphaseflow.2016.06.021.

[14] T. Yue, A. Kruisbrink, F. Pearce, H. P. Morvan, "Numerical Simulation of Jet Fragmentation in Multi-Fluid Medium Using Smoothed Particle Hydrodynamics", $9^{\text {th }}$ International SPHERIC workshop, Paris, France (2014).

[15] T. Yue, F. Pearce, A. Kruisbrink, H. P. Morvan, "Numerical simulation of two-dimensional Kelvin-Helmholtz instability using weakly compressible smoothed particle hydrodynamics", International Journal for Numerical Methods in Fluids 78 (5), pp. 283-303 (2015). DOI10.1002/fld.4017.
[16] E. Korsukova, A. Kruisbrink, H. Morvan, P. P. Cageao, K. Simmons, "Oil Scoop Simulation and Analysis using CFD and SPH", Proceding of ASME Turbo Expo 2016: Turbine Technical Converence and Exposition, Seoul, South Korea, ASME Paper No. GT2016-57554, 7B, pp. V07BT31A027 1-8

[17] W. Bo, X. Liu, J. Glimm, X. Li, “A Robust Front Tracking Method: Verification and Application to Simulation of the Primary Breakup of a Liquid Jet", SIAM Journal on Scientific Computing, 33 (4), pp. 15051524 (2011). DOI:10.1137/10079135X.

[18] S. K. Prasad, P. Sangli, O. Buyukisik, D. Pugh, "Prediction of Gas Turbine Oil Scoop Capture Efficiency", Proceeding of ASME 2014 Gas Turbine: Power for Land, Sea and Air, New Delhi, India, ASME Paper No. GTINDIA2014-8329, pp. V001T05A004 1-8.

[19] A. Prabhakar, Y. A. Abakr, K. Simmons, "Effect of Vortex Shedding on the Performance of Scoop based Lubrication Devices", Proceedings of ASME Turbo Expo 2017: Turbomachinery Technical Conference and Exposition GT2017.

[20] L. Yaguo, J. Le, L. Zhenxia, H. Jianping, "Simulation and Analysis of Oil Scoop Capture Efficiency", Proceding of ASME Turbo Expo 2018: Turbomachinery Technical Conference and Exposition, Oslo, Norway, ASME Paer No. GT2018-75989, 1 , pp. V001T01A022; 10 pages. DOI:10.1115/GT2018-75989. 